\title{
Nonlinear one-way edge-mode interactions for frequency mixing in topological photonic crystals
}

\author{
Zhihao Lan, ${ }^{*}$ Jian Wei You, ${ }^{\dagger}$ and Nicolae C. Panoiu $\oplus^{\ddagger}$ \\ Department of Electronic and Electrical Engineering, University College London, Torrington Place, London WC1E 7JE, United Kingdom
}

(Received 19 September 2019; revised manuscript received 27 March 2020; accepted 31 March 2020; published 21 April 2020)

\begin{abstract}
Topological photonics aims to utilize topological photonic bands and corresponding edge modes to implement robust light manipulation, which can be readily achieved in the linear regime of light-matter interaction. Importantly, unlike solid-state physics, the common test bed for new ideas in topological physics, topological photonics provides an ideal platform to study wave mixing and other nonlinear interactions. These are wellknown topics in classical nonlinear optics but largely unexplored in the context of topological photonics. Here, we investigate nonlinear interactions of one-way edge modes in frequency mixing processes in topological photonic crystals. We present a detailed analysis of the band topology of two-dimensional photonic crystals with hexagonal symmetry and demonstrate that nonlinear optical processes, such as second- and third-harmonic generation, can be conveniently implemented via one-way edge modes in this setup. Moreover, we demonstrate that more exotic phenomena, such as slow-light enhancement of nonlinear interactions and harmonic generation upon interaction of backward-propagating (left-handed) edge modes, can also be realized. Our work opens up new avenues towards topology-protected frequency mixing processes in photonics.
\end{abstract}

DOI: 10.1103/PhysRevB.101.155422

\section{INTRODUCTION}

One of the most important developments in condensed matter physics in the past decades is the discovery of topological insulating materials [1,2]. These materials feature gapped bulk but gapless edge modes, which propagate unidirectionally along the system edge and are immune to local disorder, thus opening a promising avenue towards robust wave manipulation protected by topology. Inspired by this development, the emerging field of topological photonics aims to extend these topology-related ideas to the realm of photonics [3-9], which holds great promise for innovative optical devices by exploiting robust, scattering-free light propagation and manipulation. As the concept of the energy band exists at the single-particle level in both condensed matter physics and photonics, the goal of realizing photonic topological insulators can be readily achieved in the linear regime of light-matter interaction. Indeed, topological phenomena of electromagnetic waves in a linear medium can be understood by mapping Maxwell equations to the Schrödinger equation $[10,11]$.

Photonics, however, has several features not present in solid-state physics. For example, optical gain and loss can be utilized to implement non-Hermitian photonics based on parity-time symmetry [12]. The recently realized topological insulator laser demonstrates the power of this new ingredient and could deepen our understanding of the interplay between nonhermiticity and topology in active optical systems $[13,14]$. Another well-known feature is the existence of nonlinearity

\footnotetext{
*z.lan@ucl.ac.uk

$\dagger$ j.you@ucl.ac.uk

${ }^{\ddagger}$ n.panoiu@ucl.ac.uk
}

in many optical materials. In fact, optical nonlinear effects play a key role in modern photonic applications, giving rise to a variety of important phenomena, including the formation of solitons, modulation and all-optical switching of optical signals, and frequency conversion for the generation of ultrashort pulses [15]. Thus one expects new physics to emerge when adding nonlinearity to photonic systems with nontrivial topological properties. Indeed, it has been shown that when a photonic topological insulator is embedded in an optical medium with Kerr nonlinearity, lattice edge solitons can arise $[16,17]$. The possibility of enhancing the conversion efficiency of harmonic generation in the presence of topological edge states has also been studied [18-20]. Moreover, traveling-wave amplifiers [21], topological sources of quantum light [22], and nonlinear control [23] and mapping [24] of photonic topological edge states have also been achieved. Despite these important advances, the feasibility of achieving nonlinear optical mixing of edge states of topological photonic crystals ( $\mathrm{PhCs}$ ) via phase matching, which is one of the most fundamental nonlinear optical processes, has not yet been explored. We would also like to highlight the key differences between our work and previous works [18-20,24] on nonlinear optics pertaining to topological edge states: the works [18-20] are based on one-dimensional (1D) systems, so that the topological edge states are nonpropagating optical modes localized at the edges of the 1D system, whereas the system investigated in [24] is 2D, i.e., the same as ours, but there is only one topological bandgap at the fundamental frequency $(\mathrm{FF})$, and thus there are no nonlinear optical interactions between topological modes.

In this work, we study nonlinear optical interactions of edge modes in topological photonic crystals, as per Fig. 1. In particular, we present a detailed study of the band topology of $2 \mathrm{D} \mathrm{PhCs}$ with hexagonal symmetry by mapping out the 
(a)

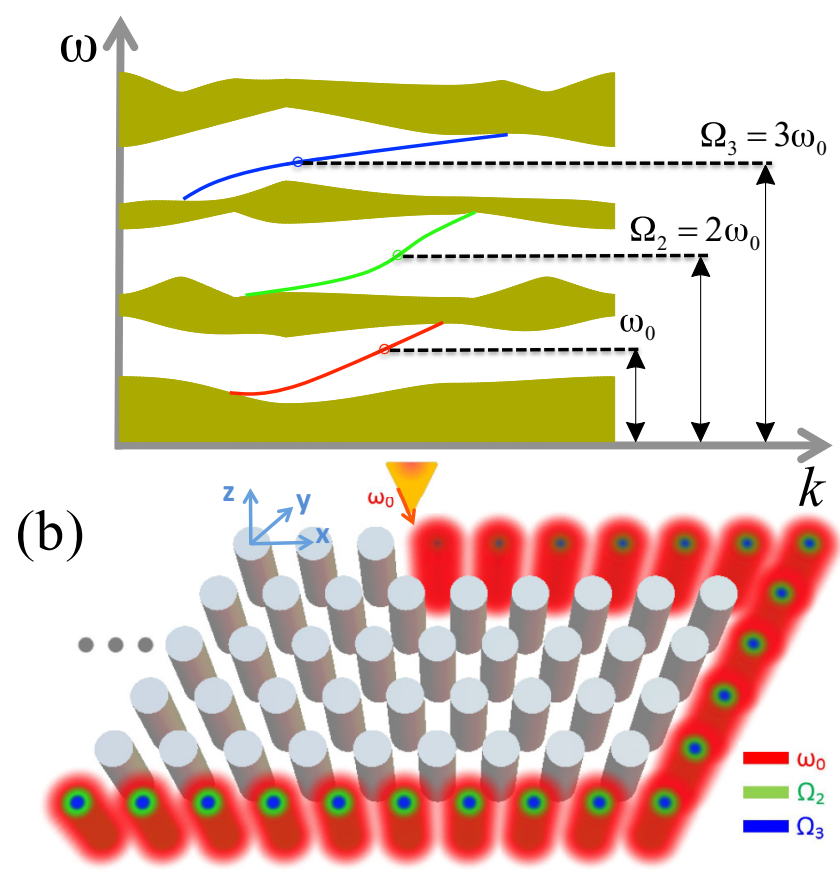

FIG. 1. (a) Schematic band structure showing the emergent edge modes due to the nontrivial topology of the bulk frequency bands. The edge modes can couple via SHG and THG frequency mixing processes. (b) Real-space illustration showing the unidirectional propagation of coupled edge modes along the system edge. The red arrow indicates the excitation source of the fundamental wave, whereas the green and blue waves are generated as a result of nonlinear wave mixing.

Chern-number-graded gap phase diagrams. Interestingly, we find that most gaps in the phase diagrams have exactly one edge state in each gap, thus providing a convenient configuration to study the nonlinear interaction of these modes. To this end, by properly tailoring the edge configuration to achieve phase matching, we show that key nonlinear optical processes, such as second- and third-harmonic generation (SHG, THG) can be readily realized in this setup. Beyond this proof-ofprinciple demonstration of these nonlinear optical processes, we further show that some more exotic nonlinear optical phenomena can also be observed in these topological PhCs, including slow-light enhanced frequency conversion efficiency and higher-harmonic generation upon interaction of so-called backward-propagating (left-handed) modes. All these novel ideas open up new avenues towards active photonic devices with novel functionalities for photonic applications.

The article is organized as follows. In the next section we present and discuss the linear optical properties of the topological PhC, whereas in Sec. III we describe the nonlinear optical interaction between one-way topological modes and the coupled-mode theory (CMT) that governs the nonlinear propagation of interacting topological modes. Moreover, in Sec. IV we briefly discuss possible experimental implementations of the ideas presented in this study, whereas in Sec. V we summarize the main conclusions of this work.

\section{LINEAR OPTICAL PROPERTIES OF THE INVESTIGATED TOPOLOGICAL PHOTONIC CRYSTAL}

In this section we describe the geometry and material parameters of the topological PhC investigated in this work, as well as the topological properties of the bulk frequency bands and edge topological modes.

\section{A. The system}

We begin by describing the system setup. In the first step, 2D PhCs possessing topological frequency gaps around frequencies of $\omega_{0}, \Omega_{2}=2 \omega_{0}$, and $\Omega_{3}=3 \omega_{0}$ are designed in order to study SHG and THG via the corresponding edge modes located inside these gaps. As such, in principle any $\mathrm{PhC}$ satisfying this condition could be employed. Nonetheless, it would be beneficial if the first gap were topological since typically the spectral separation among frequency bands and the gap widths decrease as the frequency increases. In view of this, employing the transverse magnetic modes of a $\mathrm{PhC}$ with a hexagonal symmetry lattice, which features Dirac cones at $K$ and $K^{\prime}$ points of the first Brillouin zone (FBZ) between the first and the second bands [25], is a natural choice. More specifically, one expects that for this configuration the first gap becomes topological when gapping the Dirac cones by breaking the time-reversal symmetry. Consequently, we consider triangular PhCs whose unit cell contains only one cylinder with radius $r$ as depicted in Fig. 2(a). Lattice structures with hexagonal symmetry but having more cylinders in each unit cell, like honeycomb and Kagome lattices with two and three cylinders, respectively, could potentially be employed too. The second step in our design procedure is to include magnetic and nonlinear materials. To guide potential experimental implementations and for the sake of specificity, we consider cylinders with low-permittivity $\left(\epsilon_{1}\right)$, nonmagnetic nonlinear material immersed in a magnetic background material with a high permittivity $\left(\epsilon_{2}\right)$. Note that the permittivity of the cylinders has to be lower than that of the background to ensure that Dirac cones exist.

\section{B. Topological properties of the bulk frequency bands}

We now move on to the topological properties of the bulk frequency bands of the proposed nonmagnetic $\left(\mu=\mu_{0}\right)$ PhCs whose unit cell and FBZ are shown in Figs. 2(a) and 2(b). In the following, we use the normalized frequency and momentum, $\bar{\omega}=\omega a / 2 \pi c$ and $\bar{k}=k a / \pi$, respectively, where $c$ is the speed of light and $a$ is the lattice constant. Figure 2(c) shows the photonic band structure of the PhC with $r=0.4 a$, $\epsilon_{1}=3$, and $\epsilon_{2}=18$, from which one can see the Dirac cone between the first and the second bands at $K$ and $K^{\prime}$ points. All band structures presented in this work were calculated using COMSOL MULTIPHYSICS 5.3 [26], a commercial software package based on the finite-element method, and validated using Synopsis's BandsoLvE software [27].

It is known that the Chern number of each band is 0 in systems with time-reversal symmetry [28]. A common way to break time-reversal symmetry and generate bands with a nonzero Chern number is to use magnetic materials [29-33], where the permeability tensor of the material under an external magnetic field along the $z$ axis possesses off-diagonal 
(a)
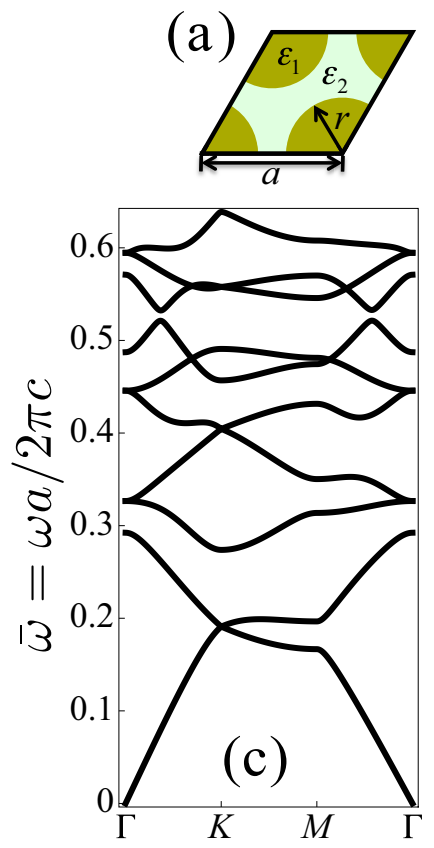

(b)
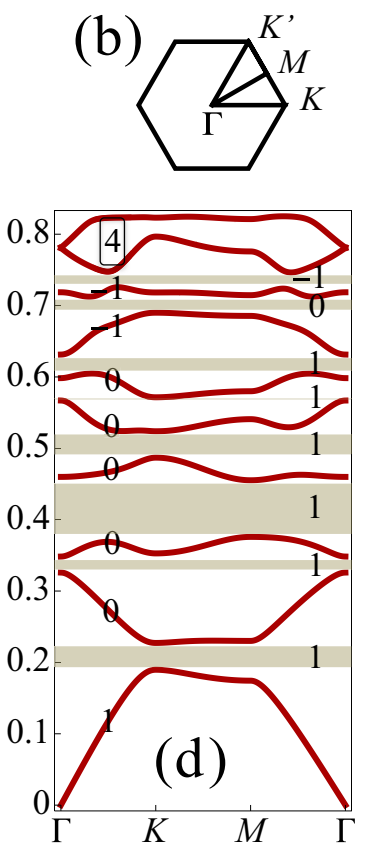

(e)
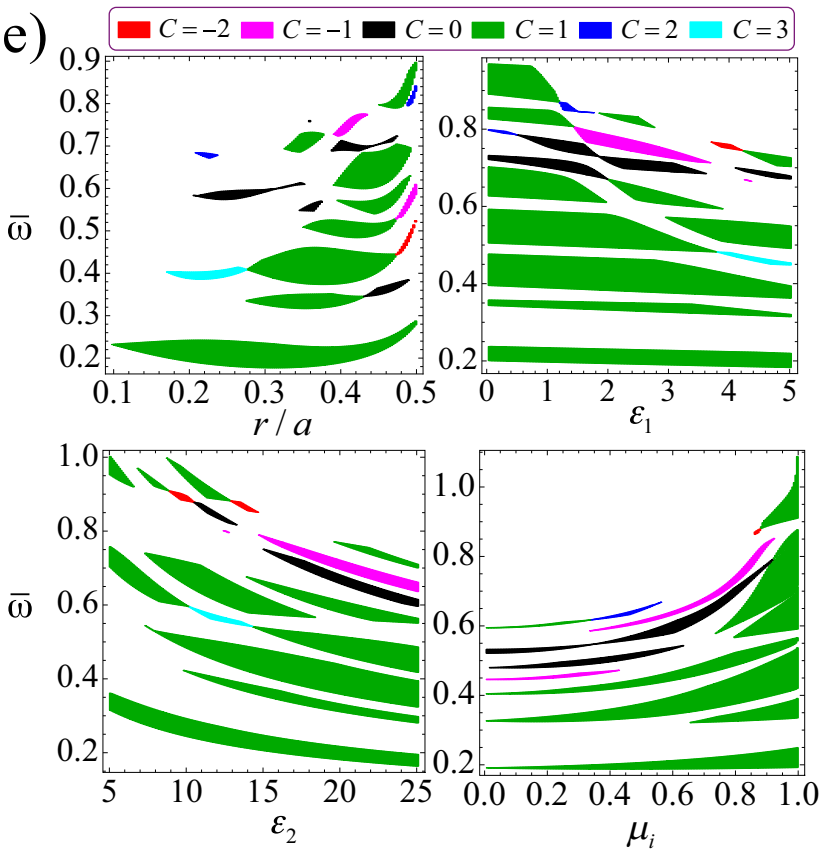

FIG. 2. (a) Unit cell with lattice constant $a$ of the $\mathrm{PhC}$, where $r$ and $\epsilon_{1}$ are the radius and relative permittivity of the cylinders, respectively, and $\epsilon_{2}$ and $\mu_{i}$ are the relative permittivity and off-diagonal component of the relative permeability of the background magnetic material, respectively. (b) The first Brillouin zone with symmetry points $\Gamma, K, K^{\prime}$, and $M$. (c) Photonic band structure of the PhC, computed for $r=0.4 a, \epsilon_{1}=3, \epsilon_{2}=18$, and $\mu_{i}=0$. (d) Nontrivial topological bands for $\mu_{i}=0.8$ [other parameters are the same as in (c)], where the Chern number of each band and the gap Chern numbers are provided (except for the last two bands, which touch each other and thus have the same Chern number). (e) Chern-number-graded gap phase diagrams when varying $r, \epsilon_{1}, \epsilon_{2}$, and $\mu_{i}$, determined for $r=0.4 a, \epsilon_{1}=3, \epsilon_{2}=18$, and $\mu_{i}=0.8$.

components in the $x-y$ plane, i.e.,

$$
\mu=\left(\begin{array}{ccc}
\mu_{0} & i \mu_{i} & 0 \\
-i \mu_{i} & \mu_{0} & 0 \\
0 & 0 & \mu_{0}
\end{array}\right)
$$

Here, we set $\mu_{0}=1$ and take $\mu_{i}$ as a parameter to quantify the effect of time-reversal symmetry breaking. Figure 2(d) shows the photonic band structure for $\mu_{i}=0.8$, where one can see that the Dirac cone is now gapped.

To characterize the topology of the frequency bands, we calculate the Chern number of the $n$th band, defined as $[3,10]$

$$
C_{n}=\frac{1}{2 \pi} \oint_{\mathrm{FBZ}} \mathcal{F}_{n}(\mathbf{k}) \cdot d \mathbf{k},
$$

where $\mathcal{A}_{n}(\mathbf{k})=\left\langle\mathbf{E}_{n}(\mathbf{k})\left|i \nabla_{\mathbf{k}}\right| \mathbf{E}_{n}(\mathbf{k})\right\rangle$ and $\mathcal{F}_{n}(\mathbf{k})=\nabla_{\mathbf{k}} \times \mathcal{A}_{n}(\mathbf{k})$ are the Berry connection and Berry curvature, respectively, with $\mathbf{E}_{n}(\mathbf{k})$ being the electric field of the $n$th band mode with momentum k. The momentum-space integral is performed over the FBZ, whereas the inner product of the Berry connection is defined as $\left\langle\mathbf{E}_{\alpha} \mid \mathbf{E}_{\beta}\right\rangle=\iint \epsilon(\mathbf{r}) \mathbf{E}_{\alpha}(\mathbf{r}) \cdot \mathbf{E}_{\beta}(\mathbf{r}) d \mathbf{r}$, with the real-space integral performed over the unit cell. The Chern number is calculated using the algorithm described in [34] (for more details about this algorithm, see Appendix A).

The calculated Chern numbers of the photonic bands are indicated in Fig. 2(d) on each band, and the gap Chern number, defined as the sum of the Chern numbers of the bands below the gap, is also given for each gap. The gap Chern number characterizes the topology of the gap in the sense that its sign determines the propagation direction of the edge modes and its value indicates the number of edge states located inside the gap [3]. An interesting feature revealed in Fig. 2(d) is that the first few gaps have Chern number $C=1$, which means that there is one edge mode in each gap and all propagate in the same direction. Therefore, this configuration provides a convenient platform to study nonlinear optical processes, e.g., SHG and THG.

To understand intuitively what regimes can be achieved with this setup, it is instructive to map out the Chern-numbergraded gap phase diagrams, defined as the variation of the gap Chern numbers with the system parameters $r, \epsilon_{1}, \epsilon_{2}$, and $\mu_{i}$. We show these gap phase diagrams in Fig. 2(e) for $r=0.4 a$, $\epsilon_{1}=3, \epsilon_{2}=18$, and $\mu_{i}=0.8$, when one parameter is varied while the others are kept fixed. The results show that most domains of the phase diagrams have $C=1$. Moreover, one can also see gaps with $C=2$ and $C=3$ and, importantly, even gaps with negative Chern numbers, $C=-1$ and $C=$ -2 . As we demonstrate, this variety of values of the gap Chern numbers leads to particularly rich physics when nonlinear interactions of topological modes are considered.

\section{Topological properties of the edge modes}

Guided by the phase diagrams in Fig. 2(e), we choose suitable parameters to create photonic gaps suitable for studying SHG and THG. According to the principle of bulk-edge correspondence in systems of a finite size, when the gap has a nonzero Chern number, one-way edge modes will emerge in the gap. We present in Fig. 3(a) the photonic band structure of 
(a)

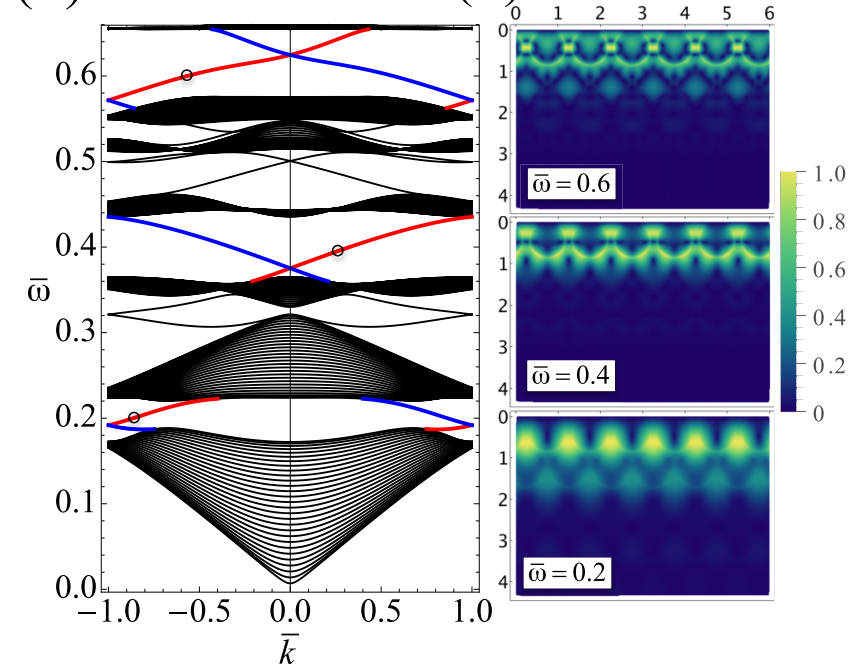

(c)
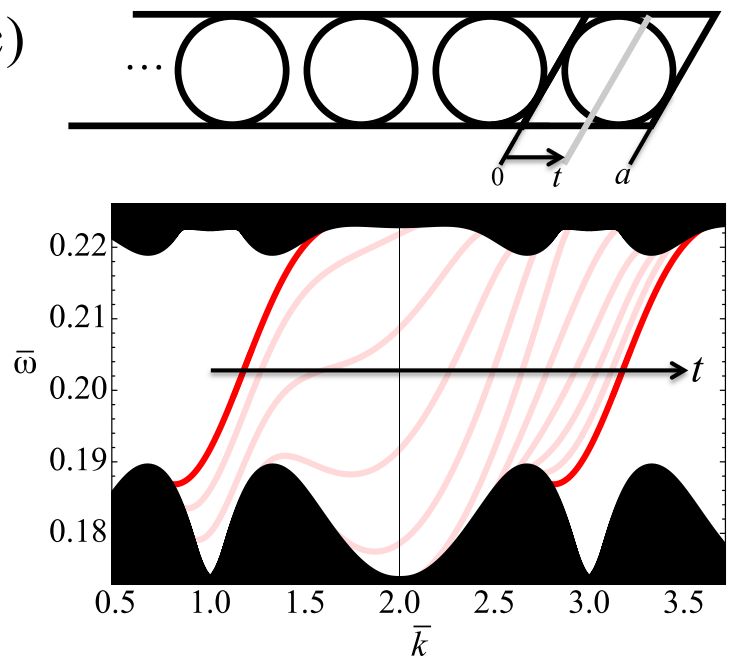

FIG. 3. (a) Photonic band structure of a 1D PhC strip that is periodic along the $x$ axis and has a finite size of 30 unit cells along the $y$ axis (top and bottom edges are terminated by a perfect electric conductor at $r=0.42 a$ ). Other simulation parameters are $\epsilon_{1}=3, \epsilon_{2}=20$, and $\mu_{i}=0.8$. Edge modes in the three gaps around $\bar{\omega}=0.2,0.4$, and 0.6 are depicted by red and blue lines and are formed at the top and bottom edges of the PhC, respectively. (b) Field profiles of the three one-way edge modes at $\bar{\omega}=0.2,0.4$, and 0.6 of the top edge. Exponential decay of the field around the $\mathrm{PhC}$ edge can be observed (integers indicate the number of unit cells). (c) Dispersion curves of edge modes can be tailored by changing the edge termination, as indicated in the sketch.

a PhC strip with 30 unit cells along the $y$ axis and periodic along the $x$ axis. This figure illustrates the emergence of various edge states across bulk photonic gaps in a range of frequencies. For the sake of clarity, we show in red and blue the edge states formed at the top and bottom edges of the PhC strip, respectively. The field profiles of the edge states at frequencies $\bar{\omega}=0.2, \bar{\Omega}_{2}=2 \bar{\omega}=0.4$, and $\bar{\Omega}_{3}=3 \bar{\omega}=0.6$, presented in Fig. 3(b), highlight the key feature of the edge state- exponential decay of the field away from the edge.

A well-known prerequisite for achieving efficient frequency conversion processes is to phase-match the interacting waves [15]. In the current context, this requires a method to tune the wave vectors of the edge modes. As far as we know this issue has not been previously discussed, perhaps due to the irrelevance of phase-matching in other (linear) physics involving edge modes. We find that the wave vector of edge modes can be readily tuned by simply changing the configuration of the edge termination. Figure 3(c) shows how the edge-mode band of the top edge changes when the location of the edge termination is varied [see the sketch at the top of Fig. 3(c)]. In general, we find that the edge-mode band shifts by about one reciprocal lattice vector, $G=2 \pi / a$, as one increases the width of the $\mathrm{PhC}$ strip by one unit cell. Note that due to the periodicity of the system along the $x$ axis, one can always shift the wave vector of the edge mode to the region of $[-\pi / a, \pi / a]$ by adding a momentum of $n G$, with $n$ a suitable integer.

\section{NONLINEAR OPTICAL INTERACTION BETWEEN ONE-WAY TOPOLOGICAL MODES}

In this section we first introduce the coupled-mode theory for SHG mediated by the topological edge modes (a similar derivation for $\mathrm{THG}$ is given in Appendix B). We then present proof-of-concept results, both numerical and analytical, on topics such as SHG and THG upon edgemode interaction, SHG in the slow-light regime, and SHG via interaction between forward- and backward-propagating edge modes.

\section{A. Coupled-mode theory describing second-harmonic generation}

The derivation of the CMT for SHG upon nonlinear interaction of topological edge modes follows the formulation of the CMT governing nonlinear pulse interactions in nonlinear PhC slab waveguides $[35,36]$. To begin with, we note that our system contains magneto-optic materials, i.e., $\hat{\mu} \neq \hat{\mu}^{T}$, but rather $\hat{\mu}=\hat{\mu}^{\dagger}$, so that we use the conjugated form of the Lorentz reciprocity theorem [37] for the vector field $\mathbf{F}=\mathbf{E}_{1}^{*} \times \mathbf{H}_{2}+\mathbf{E}_{2} \times \mathbf{H}_{1}^{*}$, where $\left\{\mathbf{E}_{1}, \mathbf{H}_{1}\right\}$ are the electric and magnetic fields of an eigenmode of the topological $\mathrm{PhC}$, whereas $\left\{\mathbf{E}_{2}, \mathbf{H}_{2}\right\}$ are the electric and magnetic fields under the effect of nonlinear polarization $\mathbf{P}_{\mathrm{NL}}$. Here, $\hat{\mu}$ is the magnetic permeability tensor and the symbols " $T$ " and " $\dagger$ " indicate the transpose and Hermitian transpose operations, respectively. Note also that as we consider $2 \mathrm{D} \mathrm{PhCs,} \mathrm{we}$ denote by $\hat{\mathbf{z}}$ the out-of-plane direction, and all the physical quantities depend only on the $x$ and $y$ coordinates.

To start with, we write the eigenmodes of the topological $\mathrm{PhC}$, at both the fundamental $(f)$ and the second-harmonic $(s)$ frequencies, denoted $\omega_{0}$ and $\Omega_{2}=2 \omega_{0}$, respectively, in their Bloch forms, i.e.,

$$
\begin{aligned}
\mathbf{E}_{1}^{f}\left(\mathbf{r}, \omega_{0}\right) & =\frac{\mathbf{e}_{f}\left(\mathbf{r}, \omega_{0}\right)}{\sqrt{P_{f}}} e^{i k_{f}\left(\omega_{0}\right) x}, \\
\mathbf{H}_{1}^{f}\left(\mathbf{r}, \omega_{0}\right) & =\frac{\mathbf{h}_{f}\left(\mathbf{r}, \omega_{0}\right)}{\sqrt{P_{f}}} e^{i k_{f}\left(\omega_{0}\right) x}
\end{aligned}
$$


and

$$
\begin{aligned}
\mathbf{E}_{1}^{s}\left(\mathbf{r}, \Omega_{2}\right) & =\frac{\mathbf{e}_{s}\left(\mathbf{r}, \Omega_{2}\right)}{\sqrt{P_{s}}} e^{i k_{s}\left(\Omega_{2}\right) x}, \\
\mathbf{H}_{1}^{s}\left(\mathbf{r}, \Omega_{2}\right) & =\frac{\mathbf{h}_{s}\left(\mathbf{r}, \Omega_{2}\right)}{\sqrt{P_{s}}} e^{i k_{s}\left(\Omega_{2}\right) x} .
\end{aligned}
$$

In these equations, $\left\{\mathbf{e}_{f}, \mathbf{h}_{f}\right\}$ and $\left\{\mathbf{e}_{s}, \mathbf{h}_{s}\right\}$ are the latticeperiodic electric and magnetic Bloch fields of the fundamental and second-harmonic waves, respectively, $k_{f, s}$ are the corresponding Bloch wave vectors, assuming that the waves propagate along the $x$ axis, and the vector $\mathbf{r}$ lies in the transverse $(x, y)$ plane. If we choose the normalization constants $P_{f, s}$ such that

$$
\frac{1}{4} \int_{-\infty}^{\infty}\left(\mathbf{e}_{f, s}^{*} \times \mathbf{h}_{f, s}+\mathbf{e}_{f, s} \times \mathbf{h}_{f, s}^{*}\right) \cdot \hat{\mathbf{x}} d y=P_{f, s},
$$

the modes $\left\{\mathbf{E}_{1}^{f}, \mathbf{H}_{1}^{f}\right\}$ and $\left\{\mathbf{E}_{1}^{s}, \mathbf{H}_{1}^{s}\right\}$ in Eqs. (3) and (4), respectively, carry $1 \mathrm{~W}$ per unit length along the longitudinal $z$ axis.

The mode power is related to the mode energy per unit length contained in one unit cell, $W$, and the group velocity, $v_{g}$, via the relation

$$
P_{f, s}=\frac{W_{f, s}}{a} v_{g}^{f, s}=\frac{W_{f, s}^{\mathrm{el}}+W_{f, s}^{\mathrm{mag}}}{a} v_{g}^{f, s},
$$

where, for nondispersive media, the electric and magnetic energies are given by the following formulas:

$$
\begin{aligned}
W_{f, s}^{\mathrm{el}} & =\frac{1}{4} \int_{A_{\mathrm{cell}}} \mathbf{e}_{f, s}^{*} \cdot \hat{\epsilon} \mathbf{e}_{f, s} d A, \\
W_{f, s}^{\mathrm{mag}} & =\frac{1}{4} \int_{A_{\text {cell }}} \mathbf{h}_{f, s}^{*} \cdot \hat{\mu} \mathbf{h}_{f, s} d A .
\end{aligned}
$$

One can also define an effective width of the mode, $w^{\text {eff }}$, in terms of the Poynting vector of the field [36],

$$
w_{f, s}^{\mathrm{eff}}=\frac{\left(\int_{-\infty}^{\infty}\left|\mathbf{e}_{f, s} \times \mathbf{h}_{f, s}\right| d y\right)^{2}}{\int_{-\infty}^{\infty}\left|\mathbf{e}_{f, s} \times \mathbf{h}_{f, s}\right|^{2} d y} .
$$

From the Maxwell equations, one can readily infer that, in the frequency domain, the optical modes satisfy the equations

$$
\begin{gathered}
\nabla \times \mathbf{E}_{1}=i \omega \hat{\mu} \mathbf{H}_{1}, \\
\nabla \times \mathbf{H}_{1}=-i \omega \hat{\epsilon} \mathbf{E}_{1},
\end{gathered}
$$

where $\omega=\omega_{0}$ and $\omega=\Omega_{2}$ for the fundamental and secondharmonic waves, respectively.

Following the CMT, for the perturbed problem, we write the electric and magnetic fields of the fundamental and second-harmonic waves as

$$
\begin{aligned}
& \mathbf{E}_{2}^{f}\left(\mathbf{r}, \omega_{0}\right)=A_{f}(x) \frac{\mathbf{e}_{f}\left(\mathbf{r}, \omega_{0}\right)}{\sqrt{P_{f}}} e^{i k_{f}\left(\omega_{0}\right) x}, \\
& \mathbf{H}_{2}^{f}\left(\mathbf{r}, \omega_{0}\right)=A_{f}(x) \frac{\mathbf{h}_{f}\left(\mathbf{r}, \omega_{0}\right)}{\sqrt{P_{f}}} e^{i k_{f}\left(\omega_{0}\right) x}
\end{aligned}
$$

and

$$
\begin{aligned}
& \mathbf{E}_{2}^{s}\left(\mathbf{r}, \Omega_{2}\right)=A_{s}(x) \frac{\mathbf{e}_{s}\left(\mathbf{r}, \Omega_{2}\right)}{\sqrt{P_{s}}} e^{i k_{s}\left(\Omega_{2}\right) x}, \\
& \mathbf{H}_{2}^{s}\left(\mathbf{r}, \Omega_{2}\right)=A_{s}(x) \frac{\mathbf{h}_{s}\left(\mathbf{r}, \Omega_{2}\right)}{\sqrt{P_{s}}} e^{i k_{s}\left(\Omega_{2}\right) x},
\end{aligned}
$$

where $A_{f}(x)$ and $A_{s}(x)$ are the slowly varying envelopes of the fundamental and second-harmonic waves, respectively, under the effect of the nonlinear polarization $\mathbf{P}_{\mathrm{NL}}$. The power per unit length carried by the fundamental and second-harmonic fields are $\left|A_{f}(x)\right|^{2}$ and $\left|A_{s}(x)\right|^{2}$, respectively, meaning that these field amplitudes are measured in $\sqrt{\mathrm{W} / \mathrm{m}}$.

The Maxwell equations for the perturbed fields are

$$
\begin{gathered}
\nabla \times \mathbf{E}_{2}=i \omega \hat{\mu} \mathbf{H}_{2}, \\
\nabla \times \mathbf{H}_{2}=-i \omega \hat{\epsilon} \mathbf{E}_{2}-i \omega \mathbf{P}_{\mathrm{NL}}^{\omega},
\end{gathered}
$$

where $\omega=\omega_{0}$ and $\omega=\Omega_{2}$ for the fundamental and secondharmonic waves, respectively. Moreover, starting from the equation for the second-order nonlinear polarization in the time domain, $\mathbf{P}_{\mathrm{NL}}(\mathbf{r}, t)=\hat{\chi}^{(2)}(\mathbf{r}, t): \mathbf{E}(\mathbf{r}, t) \mathbf{E}(\mathbf{r}, t)$, where $\hat{\chi}^{(2)}$ is the second-order susceptibility tensor, one can easily show that the nonlinear polarization at the fundamental and second-harmonic frequencies can be written as

$\mathbf{P}_{\mathrm{NL}}^{f}\left(\mathbf{r}, \omega_{0}\right)=\frac{2 A_{f}^{*}(x) A_{s}(x)}{\sqrt{P_{f} P_{s}}} \hat{\chi}^{(2)}: \mathbf{e}_{f}^{*}\left(\mathbf{r}, \omega_{0}\right) \mathbf{e}_{s}\left(\mathbf{r}, \Omega_{2}\right) e^{i\left(k_{s}-k_{f}\right) x}$,

$\mathbf{P}_{\mathrm{NL}}^{s}\left(\mathbf{r}, \Omega_{2}\right)=\frac{A_{f}^{2}(x)}{P_{f}} \hat{\chi}^{(2)}: \mathbf{e}_{f}\left(\mathbf{r}, \omega_{0}\right) \mathbf{e}_{f}\left(\mathbf{r}, \omega_{0}\right) e^{2 i k_{f} x}$.

We now use the 2D form of the divergence theorem, which states that for any general function $\mathbf{F}$,

$$
\int_{A} \nabla \cdot \mathbf{F} d A=\frac{\partial}{\partial x} \int_{A} \mathbf{F} \cdot \hat{\mathbf{x}} d A+\oint_{\partial A} \mathbf{F} \cdot \hat{\mathbf{n}} d l,
$$

where $A$ is an arbitrary cross section perpendicular to the direction of wave propagation, $\hat{\mathbf{x}}$, and $\hat{\mathbf{n}}$ is the unit vector outwardly normal onto $\partial A$ in the plane of $A$. If we take $A$ to extend to $\infty$ along the $y$ axis, the line integral vanishes for fields that decay exponentially to $\infty$. Moreover, the left-hand side (1.h.s.) of Eq. (16) can be written as

$$
\int_{-\infty}^{\infty} \nabla \cdot \mathbf{F} d y=i \omega \int_{-\infty}^{\infty} \mathbf{E}_{1}^{*} \cdot \mathbf{P}_{\mathrm{NL}}^{\omega} d y
$$

In deriving this equation, we used the vector identity $\nabla \cdot(\mathbf{A} \times$ $\mathbf{B})=\mathbf{B} \cdot(\nabla \times \mathbf{A})-\mathbf{A} \cdot(\nabla \times \mathbf{B})$ and the fact that, since $\hat{\epsilon}$ and $\hat{\mu}$ are Hermitian, the identities $\mathbf{E}_{1}^{*} \cdot \hat{\epsilon} \mathbf{E}_{2}=\left(\hat{\epsilon} \mathbf{E}_{1}\right)^{*} \cdot \mathbf{E}_{2}$ and $\mathbf{H}_{1}^{*} \cdot \hat{\mu} \mathbf{H}_{2}=\left(\hat{\mu} \mathbf{H}_{1}\right)^{*} \cdot \mathbf{H}_{2}$ hold.

Let us now consider Eq. (17), written for the fundamental frequency,

$$
\begin{aligned}
\int_{-\infty}^{\infty} \nabla \cdot \mathbf{F} d y= & \frac{2 i \omega_{0} A_{f}^{*} A_{s} e^{i \Delta k x}}{P_{f} \sqrt{P_{s}}} \\
& \times \int_{-\infty}^{\infty} \mathbf{e}_{f}^{*} \cdot \hat{\chi}^{(2)}\left(\omega_{0} ;-\omega_{0}, \Omega_{2}\right): \mathbf{e}_{f}^{*} \mathbf{e}_{s} d y,
\end{aligned}
$$


where $\Delta k=k_{s}\left(\Omega_{2}\right)-2 k_{f}\left(\omega_{0}\right)$ is the wave-vector mismatch. Moreover, the right-hand side (r.h.s.) of Eq. (16) can be cast as

$$
\begin{aligned}
& \frac{\partial}{\partial x} \int_{-\infty}^{\infty} \mathbf{F} \cdot \hat{\mathbf{x}} d y=\frac{\partial}{\partial x} \int_{-\infty}^{\infty}\left(\mathbf{E}_{1}^{f *} \times \mathbf{H}_{2}^{f}+\mathbf{E}_{2}^{f} \times \mathbf{H}_{1}^{f *}\right) \cdot \hat{\mathbf{x}} d y \\
& \quad=\frac{d A_{f}(x)}{d x} \frac{1}{P_{f}} \int_{-\infty}^{\infty}\left(\mathbf{e}_{f}^{*} \times \mathbf{h}_{f}+\mathbf{e}_{f} \times \mathbf{h}_{f}^{*}\right) \cdot \hat{\mathbf{x}} d y=4 \frac{d A_{f}(x)}{d x}
\end{aligned}
$$

Comparing Eqs. (18) and (19), we arrive at the equation describing the slowly varying mode amplitude $A_{f}(x)$,

$$
\frac{d A_{f}(x)}{d x}=i \gamma_{f}^{(2)}(x) A_{f}^{*}(x) A_{s}(x) e^{i \Delta k x}
$$

where the nonlinear coefficient at the fundamental frequency is

$$
\gamma_{f}^{(2)}(x)=\frac{\omega_{0}}{2 P_{f} \sqrt{P_{s}}} \int_{-\infty}^{\infty} \mathbf{e}_{f}^{*} \cdot \hat{\chi}^{(2)}\left(\omega_{0} ;-\omega_{0}, \Omega_{2}\right): \mathbf{e}_{f}^{*} \mathbf{e}_{s} d y .
$$

The equation governing the evolution of the slowly varying mode amplitude of the second-harmonic, $A_{s}(x)$, is derived in a similar way. Thus, when $\omega=\Omega_{2}$, the 1.h.s. of Eq. (16) becomes

$$
\begin{aligned}
\int_{-\infty}^{\infty} \nabla \cdot \mathbf{F} d y= & \frac{i \Omega_{2} A_{f}^{2} e^{-i \Delta k x}}{P_{f} \sqrt{P_{s}}} \\
& \times \int_{-\infty}^{\infty} \mathbf{e}_{s}^{*} \cdot \hat{\chi}^{(2)}\left(\Omega_{2} ; \omega_{0}, \omega_{0}\right): \mathbf{e}_{f} \mathbf{e}_{f} d y,
\end{aligned}
$$

and the r.h.s. of Eq. (16) can be expressed as

$$
\begin{aligned}
& \frac{\partial}{\partial x} \int_{-\infty}^{\infty} \mathbf{F} \cdot \hat{\mathbf{x}} d y=\frac{\partial}{\partial x} \int_{-\infty}^{\infty}\left(\mathbf{E}_{1}^{s *} \times \mathbf{H}_{2}^{s}+\mathbf{E}_{2}^{s} \times \mathbf{H}_{1}^{s *}\right) \cdot \hat{\mathbf{x}} d y \\
& \begin{array}{l}
\chi_{\mathrm{eff}, f}^{(2)}(x)=\frac{a^{2} \int_{-\infty}^{\infty} \mathbf{e}_{f}^{*} \cdot \hat{\chi}^{(2)}\left(\omega_{0} ;-\omega_{0}, \Omega_{2}\right): \mathbf{e}_{f}^{*} \mathbf{e}_{s} d y}{\int_{A_{\text {cell }}}\left(\mathbf{e}_{f}^{*} \cdot \hat{\epsilon}_{r} \mathbf{e}_{f}+Z_{0}^{2} \mathbf{h}_{f}^{*} \cdot \hat{\mu}_{r} \mathbf{h}_{f}\right) d A\left[\int_{A_{\text {cell }}}\left(\mathbf{e}_{s}^{*} \cdot \hat{\epsilon}_{r} \mathbf{e}_{s}+Z_{0}^{2} \mathbf{h}_{s}^{*} \cdot \hat{\mu}_{r} \mathbf{h}_{s}\right) d A\right]^{1 / 2}}, \\
\chi_{\mathrm{eff}, s}^{(2)}(x)=\frac{a^{2} \int_{-\infty}^{\infty} \mathbf{e}_{s}^{*} \cdot \hat{\chi}^{(2)}\left(\Omega_{2} ; \omega_{0}, \omega_{0}\right): \mathbf{e}_{f} \mathbf{e}_{f} d y}{\int_{A_{\text {cell }}}\left(\mathbf{e}_{f}^{*} \cdot \hat{\epsilon}_{r} \mathbf{e}_{f}+Z_{0}^{2} \mathbf{h}_{f}^{*} \cdot \hat{\mu}_{r} \mathbf{h}_{f}\right) d A\left[\int_{A_{\text {cell }}}\left(\mathbf{e}_{s}^{*} \cdot \hat{\epsilon}_{r} \mathbf{e}_{s}+Z_{0}^{2} \mathbf{h}_{s}^{*} \cdot \hat{\mu}_{r} \mathbf{h}_{s}\right) d A\right]^{1 / 2}} .
\end{array}
\end{aligned}
$$

$$
=\frac{d A_{s}(x)}{d x} \frac{1}{P_{s}} \int_{-\infty}^{\infty}\left(\mathbf{e}_{s}^{*} \times \mathbf{h}_{s}+\mathbf{e}_{s} \times \mathbf{h}_{s}^{*}\right) \cdot \hat{\mathbf{x}} d y=4 \frac{d A_{s}(x)}{d x} .
$$

Finally, from Eqs. (22) and (23), we obtain the governing equation for the slowly varying amplitude $A_{s}(x)$,

$$
\frac{d A_{s}(x)}{d x}=i \gamma_{s}^{(2)}(x) A_{f}^{2}(x) e^{-i \Delta k x},
$$

where the nonlinear coefficient at the second harmonic is

$$
\gamma_{s}^{(2)}(x)=\frac{\Omega_{2}}{4 P_{f} \sqrt{P_{s}}} \int_{-\infty}^{\infty} \mathbf{e}_{s}^{*} \cdot \hat{\chi}^{(2)}\left(\Omega_{2} ; \omega_{0}, \omega_{0}\right): \mathbf{e}_{f} \mathbf{e}_{f} d y .
$$

In order to better understand the effects of the slow light on the strength of the nonlinear interaction, we use Eq. (6) in conjunction with Eq. (7) to express the nonlinear coefficients as

$$
\begin{aligned}
\gamma_{f}^{(2)}(x) & =\frac{4 Z_{0}^{3 / 2} \omega_{0} n_{g, f} \sqrt{n_{g, s}}}{\sqrt{a}} \chi_{\mathrm{eff}, f}^{(2)}(x) \\
& =\frac{4 \omega_{0}}{\sqrt{a} \epsilon_{0}^{3 / 2} v_{g, f} \sqrt{v_{g, s}}} \chi_{\mathrm{eff}, f}^{(2)}(x), \\
\gamma_{s}^{(2)}(x) & =\frac{2 Z_{0}^{3 / 2} \Omega_{2} n_{g, f} \sqrt{n_{g, s}}}{\sqrt{a}} \chi_{\mathrm{eff}, s}^{(2)}(x) \\
& =\frac{2 \Omega_{2}}{\sqrt{a} \epsilon_{0}^{3 / 2} v_{g, f} \sqrt{v_{g, s}}} \chi_{\mathrm{eff}, s}^{(2)}(x),
\end{aligned}
$$

where $Z_{0}$ is the vacuum impedance, $n_{g, f / s}=c / v_{g, f / s}$ are the group indices of the two interacting modes, and the effective second-order susceptibilities $\chi_{\mathrm{eff}, f / s}^{(2)}(x)$ are defined by the
It should be noted that the nonlinear coefficients and nonlinear effective susceptibilities vary with the $x$ coordinate over a characteristic length equal to the lattice constant, $a$, whereas the characteristic length over which the field amplitudes vary is equal to $1 / \Delta k$. When the two interacting waves are nearly phase-matched, $a \ll 1 / \Delta k$. Therefore, in order to describe the nonlinear mode interactions, it is convenient to introduce the averaged physical quantities

$$
\begin{aligned}
\bar{\gamma}_{f, s}^{(2)} & =\frac{1}{a} \int_{x_{0}}^{x_{0}+a} \gamma_{f, s}^{(2)}(x) d x, \\
\bar{\chi}_{\mathrm{eff}, f / s}^{(2)} & =\frac{1}{a} \int_{x_{0}}^{x_{0}+a} \chi_{\mathrm{eff}, f / s}^{(2)}(x) d x,
\end{aligned}
$$

where $x_{0}$ is arbitrary. Then, by averaging Eqs. (20) and (24), we arrive at the system of CMT governing the nonlinear dynamics of the interacting modes,

$$
\begin{aligned}
& \frac{d \bar{A}_{f}(x)}{d x}=i \bar{\gamma}_{f}^{(2)} \bar{A}_{f}^{*}(x) \bar{A}_{s}(x) e^{i \Delta k x}, \\
& \frac{d \bar{A}_{s}(x)}{d x}=i \bar{\gamma}_{s}^{(2)} \bar{A}_{f}^{2}(x) e^{-i \Delta k x},
\end{aligned}
$$

where $\bar{A}_{f}(x)$ and $\bar{A}_{s}(x)$ are the averaged mode amplitudes at the fundamental and second-harmonic frequencies, respectively. 


\section{B. Description of the full-wave numerical simulations approach}

The full-wave dynamics of the nonlinear interaction of topological edge modes (SHG and THG) were determined numerically using the module "Electromagnetic Waves, Frequency Domain" of COMSOL MULTIPHYSICS. Thus, to simulate the nonlinear frequency mixing processes in COMSOL, we defined two "Electromagnetic Waves, Frequency Domain" models: one for the fundamental frequency $\omega_{0}$ and one for the second (third) harmonic frequency $\Omega_{2}\left(\Omega_{3}\right)$. The two models are coupled using a "Polarization" feature added to each of the models. We assumed that for both the SHG and the THG cases the nonlinear susceptibilities are diagonal tensors, the diagonal elements being $\chi_{2}$ and $\chi_{3}$, respectively.

For the study of SHG (where we consider transversemagnetic-polarized modes), the nonlinear polarizations at the $\mathrm{FF}$ and second harmonic ( $\mathrm{SH})$ are

$$
\begin{aligned}
& P_{\mathrm{NL}, z}^{\omega_{0}}=2 \chi_{2} E_{2 z} E_{1 z}^{*}, \\
& P_{\mathrm{NL}, z}^{\Omega_{2}}=\chi_{2} E_{1 z}^{2} .
\end{aligned}
$$

For the study of THG, the corresponding nonlinear polarizations are

$$
\begin{aligned}
& P_{\mathrm{NL}, z}^{\omega_{0}}=3 \chi_{3} E_{3 z} E_{1 z}^{* 2}, \\
& P_{\mathrm{NL}, z}^{\Omega_{3}}=\chi_{3} E_{1 z}^{3} .
\end{aligned}
$$

\section{Second-harmonic generation and third-harmonic generation upon edge-mode interaction}

We now investigate nonlinear frequency conversion processes via the edge modes indicated in Fig. 3(a) using fullwave numerical simulations of Maxwell equations with the results summarized in Fig. 4. In the following, we mainly focus on the discussion of SHG, as the results of THG can be understood similarly.

We consider cylinders made of homogeneous and isotropic nonlinear material with a scalar nonlinear second-order susceptibility of $\chi^{(2)}=10^{-21} \mathrm{C} \mathrm{V}^{-2}$ (the typical value of $\chi^{(2)}$ varies from $10^{-24}$ to $10^{-21} \mathrm{C} \mathrm{V}^{-2}$ [38]). The pump electric field $\mathbf{E}_{1}$ is induced by an external source; $\mathbf{E}_{2}$, by the nonlinear polarization at the $\mathrm{SH}$, generated by $\mathbf{E}_{1}$. The amplitude of $\mathbf{E}_{1}$ is chosen such that the undepleted pump approximation holds, i.e., the amplitude of $\mathbf{E}_{1}$ is much larger than that of $\mathbf{E}_{2}$ and thus $\mathbf{E}_{1}$ is roughly constant during the frequency conversion process. Note, however, that our analysis remains valid when this condition is not fulfilled also, our choice being chiefly guided by a more facile comparison between numerical and theoretical results, which is possible in this propagation regime.

From Figs. 4(a) and 4(b), one can observe that the field profiles of $\mathbf{E}_{1}$ and $\mathbf{E}_{2}$ are indeed the same as the profiles of the edge modes shown in Fig. 3(b), indicating that the two edge modes are indeed nonlinearly interacting via the SHG-a key result of our work. The physics of this nonlinear process can be accurately captured by the CMT (see Appendix C). In particular, the period of spatial oscillations of the $\mathrm{SH}$ field $\mathbf{E}_{2}$ is determined by the wave-vector mismatch $\Delta k=k_{\mathrm{SH}}-2 k_{\mathrm{FF}}$ [ $\Delta k=k_{\mathrm{TH}}-3 k_{\mathrm{FF}}$ for third-harmonic (TH) generation]. As a result, we can straightforwardly compare the numerically extracted oscillation period of $\mathbf{E}_{2}$ with the theoretical prediction of $\Lambda_{2}=2 \pi / \Delta \vec{k}$, thus confirming that the key physics of nonlinear frequency conversion processes is validated by our simulations.

We further validate these conclusions using a much larger simulation domain, with the corresponding results being presented in Figs. 4(d)-4(f). In Fig. 4(d), where $\bar{\omega}_{0}=0.2$, $\bar{\Omega}_{2}=0.4$, and $\Delta \bar{k}=0.054$, we have $\Lambda_{2}=37$. The agreement between the predictions of the CMT and direct numerical simulations, for both the period and the amplitude of $\mathrm{SH}$ power oscillation along the propagation distance, is excellent. We calculate $\Delta \bar{k}$ for all the frequencies of the interacting edge modes in Fig. 4(e) and present the theoretically calculated and numerically extracted oscillation period $\Lambda_{2}$ in Fig. 4(f). An excellent agreement between the two results can be observed, which confirms the key physics of SHG, namely, phase-matching is indeed at work in our photonic system and SHG purely via nonlinear interaction of edge modes occurs in our setup. We also confirm the edge-mode-mediated THG as shown in Figs. 4(g)-4(i), where the discrepancy in Fig. 4(i) between the numerical and the theoretical results is due to inherent limitations of numerical simulations at very small $\Delta k$.

While the SHG and THG of one-way edge modes are governed by the usual mechanism of topological protection from the chiral nature of the edge modes, where the modes can bypass structural defects, such as the sharp bends shown in Figs. 4(a)-4(c), without undergoing backscattering, the effect of structural defects on the coherence properties of nonlinear processes is an important but less understood problem. In order to answer this key question, and taking SHG as an example, we introduce a structural defect at a certain location along the edge where the fundamental and second-harmonic modes copropagate and present the corresponding simulation results in Fig. 4(j). Thus, it can be seen that, as expected, both the fundamental and the second-harmonic modes bypass the defect without experiencing backscattering. More importantly, by comparing the results in Figs. 4(j) and 4(d), one can infer that the coherence length of the SHG, namely, the oscillation period of the amplitude of the interacting modes, is not altered by the interaction with the structural defect. In other words, the coherence of the nonlinear interaction is preserved in the presence of defects, meaning that the phasematched nature of the nonlinear mode interaction process is immediately regenerated after the mode interaction with each defect. As the coherence length crucially determines the conversion efficiency of the nonlinear processes, topological protection of the coherence length in nonlinear frequency mixing processes demonstrates a new area where topology can boost the performance of photonic devices based on nonlinear optical processes.

Another relevant question, which we do not intend to fully answer here, is how other types of perturbations affect the phase-matching condition of the nonlinear optical interaction of topological modes. From a practical point of view, the most relevant such perturbation is structural disorder, and in this context we consider two cases, namely, weak and strong disorder. The case of weak disorder can be analyzed using perturbative methods. Thus, let us assume that in the absence of disorder the frequency-dependent wave-vector 
mismatch, $\Delta k_{i}(\omega)$, vanishes at a certain frequency $\omega_{0}$, that is, $\Delta k_{i}\left(\omega_{0}\right)=0$. Then, adding weak disorder to the unperturbed system will change the modal dispersion of the interacting modes, such that the wave-vector mismatch varies, say, by a small quantity, $\delta k(\omega)$. Furthermore, since one expects that $\delta k(\omega)$ has a constant sign around the frequency $\omega_{0}$, it can be seen that there is a frequency $\omega_{0}+\delta \omega_{0}$ at which the wave-vector mismatch in the presence of disorder, $\Delta k_{f}(\omega)=$ $\Delta k_{i}(\omega)+\delta k(\omega)$, vanishes, that is, $\Delta k_{f}\left(\omega_{0}+\delta \omega_{0}\right)=0$. To validate this argument, one would have to employ full-wave simulations of the nonlinear optical interaction of topological modes and average the results over an ensemble of disorder realizations large enough to achieve convergence of the ensemble average. This computational analysis would be rather unfeasible, as just a single full-wave simulation requires several days to complete. On the other hand, for large values of disorder strength, concepts such as opti- cal modes, topological properties, and photonic bandgaps cease to exist, and therefore we do not investigate this case further.

\section{Second-harmonic generation in the slow-light regime}

The slow-light regime, characterized by a significantly reduced group velocity, $v_{g}=d \omega / d k$, can be particularly effective in enhancing the efficiency of nonlinear wave interactions. In the context of SHG, this can be achieved when $v_{g}$ is reduced at one or both interacting waves. As Fig. 3(c) suggests, when one varies the location of the edge termination, the shift of the dispersion curve of the edge modes is accompanied by a change in its shape. For example, we find that for $t=$ $0.24 a$, the dispersion curve of the edge mode at the FF has a plateau [see Fig. 5(a)], leading to a peak of the group index, $n_{g}=c / v_{g}$, at $\bar{\omega}_{g}$ (see Fig. 5(b), where we define the slow-light regime by the condition $n_{g}>20$ [36]). On the other

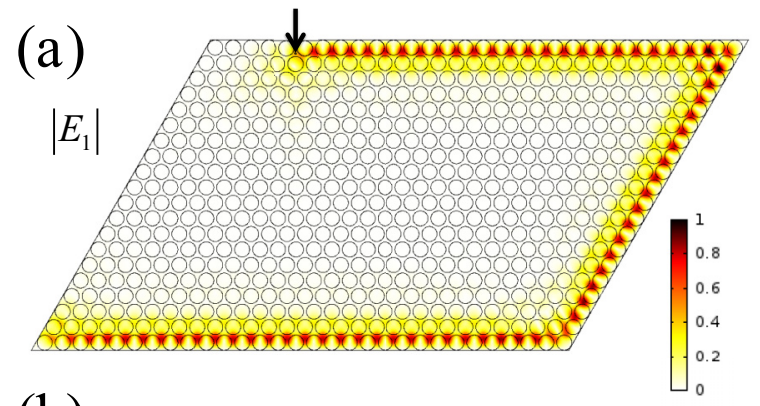

(b)

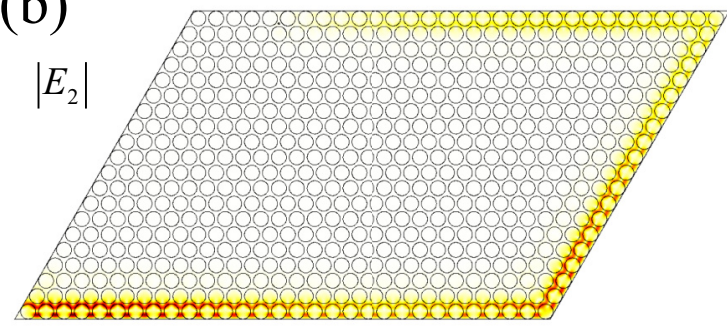

(c)

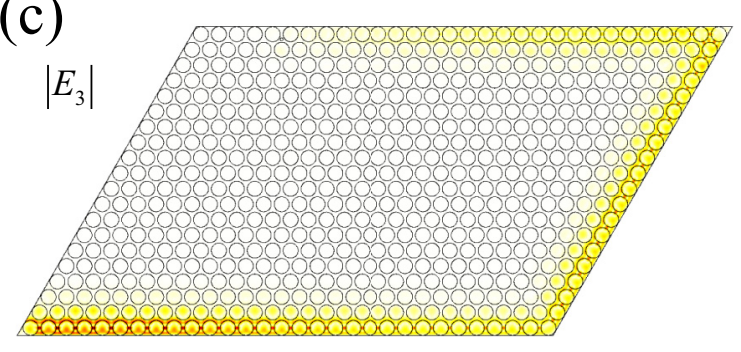

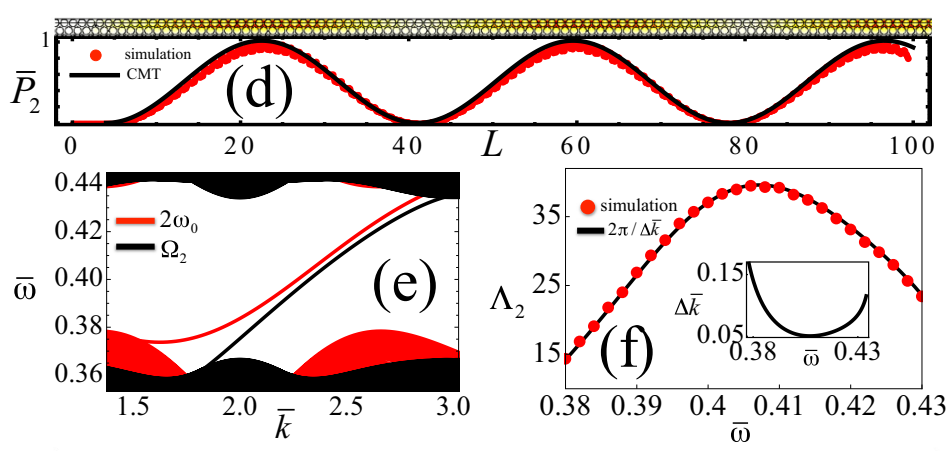

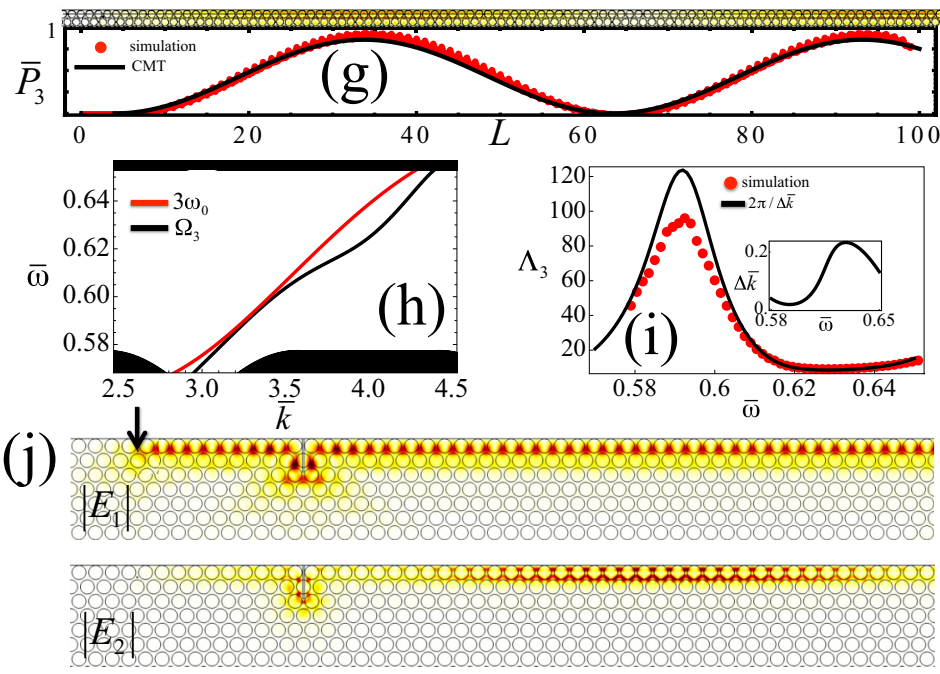

FIG. 4. (a-c) Simulated field profile intensities of $\mathbf{E}_{1}, \mathbf{E}_{2}$, and $\mathbf{E}_{3}$ at $\bar{\omega}=0.2, \bar{\Omega}_{2}=2 \bar{\omega}=0.4$, and $\bar{\Omega}_{3}=3 \bar{\omega}=0.6$, respectively, where $\mathbf{E}_{1}$ is induced by an external source indicated by an arrow, whereas $\mathbf{E}_{2}$ and $\mathbf{E}_{3}$ are generated by the corresponding nonlinear polarizations. SHG and THG are simulated separately with $\chi^{(2)}=10^{-21} \mathrm{C} \mathrm{V}^{-2}$ and $\chi^{(3)}=10^{-30} \mathrm{C} \mathrm{m} \mathrm{V}^{-3}$. In the simulations, the absorbing boundary condition $(\mathrm{ABC})$ is used for the left edge and PEC for the other edges. (d-f) Detailed analysis of SHG, where (d) shows the field intensity profile and power $\bar{P}_{2}$ (normalized by its peak value) at SH in a much larger simulation domain so as to resolve the oscillations of $\bar{P}_{2}$ due to the small phase mismatch $\Delta \bar{k}$ of the edge modes at FF $\bar{\omega}=0.2$ and SH $\bar{\Omega}_{2}=0.4$ (dotted and solid lines correspond to full numerical simulations and CMT, respectively); (e) shows the edge modes at FF $\omega_{0}$ (plotted in terms of $2 \omega_{0}$ ) and, for SHG, $\Omega_{2}$; and (f) shows the effect of phase matching, where the theoretically calculated $\Lambda_{2}=2 \pi / \Delta \bar{k}$ and numerically extracted oscillation period of $\mathbf{E}_{2}$ are in excellent agreement. The $\bar{\omega}(\Delta \bar{k})$ curve is calculated according to the edge modes presented in (e). (g-i) Results corresponding to (d)-(f), respectively, but calculated for the THG. (j) Illustration of the effect of a structural defect (simulated as the thin PEC rectangle) on the SHG interaction of topologically protected modes. Compared to the defect-free case shown in (d), one can see that the oscillation period of the amplitude of the second-harmonic mode after bypassing the defect is the same as that in $(\mathrm{d})$. 


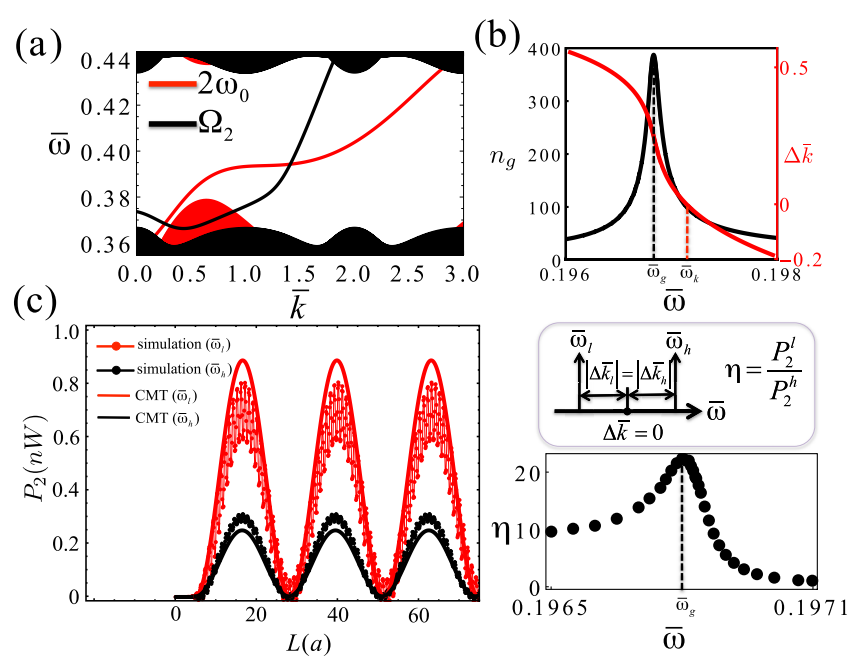

FIG. 5. (a) Dispersion of edge modes at $\omega_{0}$ and $\Omega_{2}$ determined for $t=0.24 a$, as in Fig. 3(c), whereas the other simulation parameters are the same as in Fig. 4. (b) The dispersion curve of the FF mode now shows a plateau leading to a peak of $n_{g}$ at $\bar{\omega}_{g}=0.1968$. The frequency of the phase-matching point $\bar{\omega}_{k} \equiv \bar{\omega}(\Delta \bar{k}=0)$ is $\bar{\omega}_{k}=$ 0.1972. (c) Enhancement of the SH conversion efficiency due to the slow-light effect. Left panel: An example where the SH power $P_{2}$ at two frequencies, $\bar{\omega}_{l}=0.1960$ and $\bar{\omega}_{h}=0.1975$, shows the same oscillation period, yet its oscillation amplitude at $\bar{\omega}_{l}$ (closer to the maximum of $n_{g}$, located at $\bar{\omega}_{g}$ ) is enhanced compared to that at $\bar{\omega}_{h}$. Right panels: Schematics of the formal definition of the enhancement factor $\eta$ (top) and its frequency dependence in the slow-light regime (bottom).

hand, the fact that the phase-matching spectral point $\bar{\omega}_{k} \equiv$ $\bar{\omega}(\Delta \bar{k}=0) \neq \bar{\omega}_{g}$ provides a valuable approach to study of the interplay between slow-light and phase-matching effects in the enhancement of SH conversion efficiency.

To this end, we fix the power of the FF at $P_{1}=1 \mathrm{~W}$ and compare the conversion efficiencies at two frequencies, $\bar{\omega}_{l}$ and $\bar{\omega}_{h}$, lower and higher compared to $\bar{\omega}_{k}$, respectively, chosen such that $\Delta \bar{k}\left(\bar{\omega}_{l}\right)=-\Delta \bar{k}\left(\bar{\omega}_{h}\right)$ [see the sketch in Fig. 5(c)]. As such, phase-matching has the same influence on the wave interaction in the two cases and the conversion efficiency enhancement, $\eta=P_{2}\left(\bar{\omega}_{l}\right) / P_{2}\left(\bar{\omega}_{h}\right)$, is purely due to slow-light effects. The results in Fig. 5(c) show that slow-light contribution to $\eta$ can be larger than $20 \times$. Alternative scenarios to enhance the SH conversion efficiency can also be devised, e.g., by using PhCs for which $\bar{\omega}_{k}=\bar{\omega}_{g}$ or PhCs for which the edge modes at both the $\mathrm{FF}$ and the $\mathrm{SH}$ are slow-light edge modes.

\section{E. Second-harmonic generation via interaction between forward- and backward-propagating edge modes}

We now move on to an important class of nonlinear processes, which are challenging to achieve in regular optical media, and demonstrate SHG via interaction of backwardpropagating edge modes. To this end, we exploit the existence of photonic gaps with a negative Chern number in our system, as per Fig. 2(e). In particular, we explore a case where the gap at the FF has $C=1$, while the gap at the $\mathrm{SH}$ has $C=$ -1 . The corresponding edge modes are shown in Fig. 6(c),
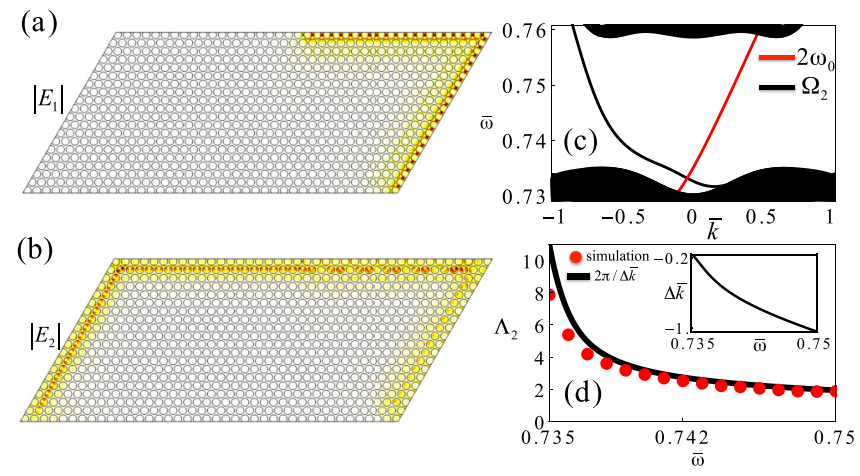

FIG. 6. SHG via interaction between forward- and backwardpropagating edge modes. This setting exploits the existence of a gap with a negative Chern number at the SH for $r=0.41 a, \epsilon_{1}=3, \epsilon_{2}=$ $20, \mu_{i}=0.82$ [see Fig. 2(e)] and edge termination at $t=0.82 a$ [see Fig. 3(c)]. (a, b) Field intensity profiles of $\mathbf{E}_{1}$ and $\mathbf{E}_{2}$ calculated for $2 \bar{\omega}_{0}=0.74$, illustrating that whereas the mode at the FF propagates clockwise $\left(C=1\right.$ for the $\omega_{0}$ gap), the edge mode at the $\mathrm{SH}$ is backward propagating ( $C=-1$ for the $\Omega_{2}$ gap). In the simulation, $\mathrm{ABC}$ is used for the bottom edge and PEC boundaries for the other edges. (c) Edge states of the two gaps, which show the hallmark of edge modes with a negative Chern number: the slope of the dispersion curve of the edge mode at the $\mathrm{SH}$ is negative. (d) Comparison between the theoretically calculated and the numerically extracted oscillation period $\Lambda_{2}$, confirming that the phase-matching mechanism is involved in this unusual nonlinear interaction regime.

which illustrates the signature of modes with a negative Chern number, i.e., the slope of the mode dispersion curve (at $\Omega_{2}$ ) is negative. The simulation results of the field profiles are presented in Figs. 6(a) and 6(b). It clearly shows that whereas the mode at the FF propagates clockwise, as it is a forwardpropagating mode, the $\mathrm{SH}$ wave propagates counterclockwise because in the left-half region of the simulation domain there is no field at the FF and consequently no nonlinear polarization at the $\mathrm{SH}$ (note that we placed the source of the FF wave at the middle of the top edge and used the absorbing boundary condition for the bottom edge). We also confirm that the phase-matching mechanism is involved in this unusual mode interaction regime, as per the results in Fig. 6(d). This backward-propagating mode regime is promising for practical applications where one is required to separate the FF mode from the mode generated at the $\mathrm{SH}$.

\section{EXPERIMENTAL CONSIDERATIONS}

The key idea of this work involves coupling the edge states within different topological frequency bandgaps via optical nonlinearity. This central idea of our study is rather general and can potentially be implemented in different experimental platforms available for topological photonics. In this section, for the sake of completeness, we first present a set of materials and parameters that can be used to experimentally implement our model system. Then we discuss further possible experimental platforms that can be used to implement the theoretic ideas and results presented in our work.

Since we separate the magnetic and nonlinear material components as the background and cylindrical regions of 
the $\mathrm{PhC}$, respectively, possible experimental implementations could be readily conceived considering the fact that at microwave frequencies, magnetic materials to realize topological bandgaps [29-33] and nonlinear materials to realize frequency mixing [39-42] are routinely used. For example, to demonstrate the topology-protected SHG, one can use as the background medium yttrium iron garnet (YIG) [43], a magnetic material widely used in recent experiments in topological photonics [29-33]. This material has relative permittivity $\epsilon=15$ and a saturation magnetization of $4 \pi M_{s}=1780 \mathrm{G}$. At a frequency of $10 \mathrm{GHz}$ and an external magnetic field of $H_{0}=1000$ Oe along the $z$ axis, the components of the permeability of YIG in the $x-y$ plane are $\mu_{\text {diag }}=0.85$ and $\mu_{\text {off-diag }}=0.54$. For the nonlinear material, one could use $\mathrm{NaNO}_{2}$, which has relative permittivity $\epsilon=4.18$ and $\chi^{(2)}=$ $3.2 \times 10^{-22} \mathrm{C} \mathrm{V}^{-2}$ frequency of tens of $\mathrm{GHz}$ [39]. Setting the radius of the cylinders to $r=0.35 a$, with $a \approx 3 \mathrm{~mm}$, the system has topological bandgaps at $\bar{\omega}_{0} \in[0.195,0.23]$ and $\bar{\Omega}_{2} \in[0.385,0.41]$, so that SHG is achieved in the frequency interval of $\bar{\omega}_{0} \in[0.195,0.205]$. The effects of material losses and frequency dispersion in a ferrite at microwave frequencies have been discussed in Ref. [28], showing that for YIG, the decay length is around $1300 a$, thus far exceeding practical structural dimensions, and the bandgap width slightly decreases, by about $6 \%$.

Furthermore, recent advances in different experimental platforms for topological photonics [3-9] provide a variety of choices available to implement the idea of topologically protected nonlinear frequency mixing processes in diverse photonic systems. More specifically, as nonlinearity exists in many optical materials and is easy to incorporate in an experimental platform, the key task reduces to designing photonic systems with several topological frequency bandgaps that can satisfy the frequency and phase-matching conditions. Regarding this requirement, we stress that several recent experiments have demonstrated the existence of topological bandgaps using coupled waveguides or resonators [44-46]. In these tight-binding systems, further topological bandgaps at higher frequencies can readily be created by considering waveguide or resonance modes at higher frequencies.

In what follows, we outline and briefly discuss several such experimental configurations in which this can potentially be achieved.

(i) Nonlinear and magnetic metamaterials. Magnetism and nonlinearity can be easily implemented using metamaterials [47-50], which provides an alternative to using regular materials, such as YIG and $\mathrm{NaNO}_{2}$, suggested above. For example, to create nonlinear metamaterials, approaches such as insertion of nonlinear elements, nonlinear host medium, and nonlinear transmission lines are commonly used [49]. Artificial magnetism at optical frequencies can be created using metamaterials based on split-ring resonators [47]. Harmonic generation and topological edge states have also been studied in such types of metamaterials [48,50].

(ii) Graphene plasmonic crystals [51-54]. In Ref. [52] (see Fig. 3(c) therein) the existence of topological edge modes within different bandgaps of a graphene plasmonic crystal has been demonstrated. Moreover, in another study [51] (see Fig. 2(a) therein) the existence of two Dirac points at the frequencies of 3.4 and $6.8 \mathrm{THz}$ has been demonstrated. These
Dirac points can be gapped out to form two topological bandgaps (ideal for SHG) under an external magnetic field as shown in [52] and [53]. In fact, recently, we have studied the topologically protected four-wave mixing process in a graphene metasurface [54].

(iii) Mimicking time-reversal breaking and synthesizing magnetic fields for photons [44,45,55]. Using an array of evanescently coupled helical waveguides, topological oneway edge states similar to that presented in Fig. 3 can be created without the need for an external magnetic field (see, e.g., Fig. 2(b) in Ref. [44]). Alternatively, one can also create one-way edge states using synthetic magnetic fields rather than a real magnetic field in an array of coupled opticalring resonators (e.g., see Fig. 4 in Ref. [45]). Furthermore, additional topological bandgaps at higher frequencies can be readily created by considering waveguide modes at corresponding frequencies.

(iv) Photonic quantum valley [46,56-58] or spin [24,5964] Hall (QVH or QSH) crystals with time-reversal symmetry. Recent experiments on valley-Hall-like photonic systems have demonstrated the existence of one [46,56,57] or two [58] (see Fig. 2(b) therein) topological bandgaps and corresponding edge modes within the gaps. As for photonic QSH systems, the experiments in [24], [60], and [61] have demonstrated the existence of one topological bandgap. Moreover, the proposal in [64] (see Figs. 3 and 4 therein) using optically passive elements to realize the optical version of the QSH insulator has shown the existence of multiple topological bandgaps.

\section{CONCLUSIONS}

In this work, we have demonstrated topology-protected nonlinear frequency conversion processes via one-way edge modes of topological photonic crystals. Apart from the proofof-concept implementations, such as SHG and THG, we also showed that more complex behaviors, such as slow-light effects and counter-propagating mode interaction, can also be realized within the setup. A special aspect of nonlinear processes, i.e., the phase-matching condition, requires a new level of control of the edge modes, which has not been discussed previously. This condition requires a method to tune the dispersion of the edge modes, which we found can be conveniently achieved by tailoring the geometry of the edge termination. Our work reveals that the coherence length characterizing the nonlinear optical interactions considered in this study, which crucially determines the efficiency of the topologically protected nonlinear frequency mixing processes, is robust against structural defects. Our proposed setup provides a platform for studying additional phenomena, e.g., when the frequency gap has a large Chern number $(|C| \neq 0,1$ [65]) one can explore how to excite one of the several edge modes in the gap or how to couple edge modes belonging to different gaps via the nonlinearity of the medium.

Importantly, nonlinear interactions of topological modes, such as sum- and difference-frequency generation, highharmonic generation, and four-wave mixing, can be readily implemented within our setup. Our work may also stimulate the search for other lattice geometries or setups where one can optimize the gap properties for specific applications. For example, in the Chern number graded gap phase diagrams 
in Fig. 2(e), apart from the gap of $C=1$, other gaps with $C=-1,-2,2$, and 3 are typically narrow and appear at high frequencies, so designing setups where these gaps are wide and are formed at low frequencies is particularly relevant from the experimental point of view. Beyond the experimental implementation of our model system at microwave frequencies using magnetic and nonlinear materials, we also discussed several different possible implementations using diverse experimental platforms available for topologic photonics. Last but not least, the concept of topology-protected nonlinear frequency mixing is very general in that it applies not only to photonics, but also to plasmonics $[52,53,66,67]$, phononics [68-70], magnonics [71-73], and exciton-polariton systems [74-77], thus we expect that our work will have a broad impact.

\section{ACKNOWLEDGMENTS}

This work was supported by the European Research Council (ERC; Grant No. ERC-2014-CoG- 648328). We acknowledge the use of the UCL Legion High Performance Computing Facility (Legion@UCL) and associated support services in the completion of this work.

\section{APPENDIX A: COMPUTATION OF CHERN NUMBERS}

The Chern number of the $n$th band of the photonic crystal is defined by Eq. (2) based on Refs. [3] and [10]. An efficient numerical algorithm to compute it was introduced in Ref. [34]. In this method, from the eigenmode $\left|\mathbf{E}_{n}(\mathbf{k})\right\rangle$ of the $n$th band, which can be accessed directly from the eigenfrequency solver of COMSOL, one can define a $U(1)$ link variable,

$$
U_{\alpha}\left(\mathbf{k}_{l}\right)=\frac{\left\langle\mathbf{E}_{n}\left(\mathbf{k}_{l}\right) \mid \mathbf{E}_{n}\left(\mathbf{k}_{l}+\mathbf{e}_{\alpha}\right)\right\rangle}{\left|\left\langle\mathbf{E}_{n}\left(\mathbf{k}_{l}\right) \mid \mathbf{E}_{n}\left(\mathbf{k}_{l}+\mathbf{e}_{\alpha}\right)\right\rangle\right|},
$$

where $\mathbf{k}_{l}$ is a lattice point in the discretized Brillouin zone and $\mathbf{e}_{\alpha}$ is the lattice displacement in the direction $\alpha(\alpha=1,2)$. Furthermore, a lattice field strength can be defined by the link variable,

$$
F_{12}\left(\mathbf{k}_{l}\right)=\ln \left[U_{1}\left(\mathbf{k}_{l}\right) U_{2}\left(\mathbf{k}_{l}+\mathbf{e}_{1}\right) U_{1}^{-1}\left(\mathbf{k}_{l}+\mathbf{e}_{2}\right) U_{2}^{-1}\left(\mathbf{k}_{l}\right)\right],
$$

where the lattice field strength is defined as the principal branch of the logarithm $-\pi<F_{12}\left(\left(\mathbf{k}_{l}\right) / i \leqslant \pi\right.$. Finally, the Chern number of the $n$th band can be calculated from the lattice field strength according to

$$
C_{n}=\frac{1}{2 \pi i} \sum_{l} F_{12}\left(\mathbf{k}_{l}\right),
$$

where the sum is taken over all the lattice points in the discretized Brillouin zone. The $C_{n}$ defined above is manifestly gauge invariant and strictly an integer for arbitrary lattice parameters. This is because if we introduce a gauge potential

$$
A_{\alpha}\left(\mathbf{k}_{l}\right)=\ln U_{\alpha}\left(\mathbf{k}_{l}\right), \quad-\pi<A_{\alpha}\left(\mathbf{k}_{l}\right) / i \leqslant \pi,
$$

we can get

$$
F_{12}\left(\mathbf{k}_{l}\right)=\Delta_{1} A_{2}\left(\mathbf{k}_{l}\right)-\Delta_{2} A_{1}\left(\mathbf{k}_{l}\right)+i 2 \pi n_{12}\left(\mathbf{k}_{l}\right),
$$

where $\Delta_{\alpha} f\left(\mathbf{k}_{l}\right)=f\left(\mathbf{k}_{l}+\mathbf{e}_{\alpha}\right)-f\left(\mathbf{k}_{l}\right)$ and $n_{12}\left(\mathbf{k}_{l}\right)$ is an integer-valued field, chosen in such a way that $F_{12}\left(\mathbf{k}_{l}\right) / i$ takes a value within the principal branch. Consequently, we can get

$$
C_{n}=\sum_{l} n_{12}\left(\mathbf{k}_{l}\right),
$$

which shows that $C_{n}$ is an integer. Certainly, this does not mean that any coarse discretization of the FBZ will ensure a converged Chern number; nevertheless, asymptotic convergence of the Chern number requires only a moderately dense discretization. In our calculations of the data presented in Fig. 2(e), we find that a $20 \times 20$ discretization of the FBZ suffices.

\section{APPENDIX B: COUPLED-MODE THEORY FOR THIRD-HARMONIC GENERATION}

The coupled-mode equations for third-harmonic generation can be derived in a way similar to that for secondharmonic generation presented in Sec. III A. Here we outline the main steps $[35,36]$. First, one can write down the expressions for the fundamental $(f)$ and third $(t)$ harmonic waves, $\left(\mathbf{E}_{1}^{f}, \mathbf{H}_{1}^{f}, \mathbf{E}_{2}^{f}, \mathbf{H}_{2}^{f}, \mathbf{P}_{\mathrm{NL}}^{f}\right)$ and $\left(\mathbf{E}_{1}^{t}, \mathbf{H}_{1}^{t}, \mathbf{E}_{2}^{t}, \mathbf{H}_{2}^{t}, \mathbf{P}_{\mathrm{NL}}^{t}\right)$, in the Bloch forms

$$
\begin{aligned}
\mathbf{E}_{1}^{f}\left(\mathbf{r}, \omega_{0}\right) & =\frac{\mathbf{e}_{f}\left(\mathbf{r}, \omega_{0}\right)}{\sqrt{P_{f}}} e^{i k_{f}\left(\omega_{0}\right) x} \\
\mathbf{H}_{1}^{f}\left(\mathbf{r}, \omega_{0}\right) & =\frac{\mathbf{h}_{f}\left(\mathbf{r}, \omega_{0}\right)}{\sqrt{P_{f}}} e^{i k_{f}\left(\omega_{0}\right) x} \\
\mathbf{E}_{1}^{t}\left(\mathbf{r}, \Omega_{3}\right) & =\frac{\mathbf{e}_{t}\left(\mathbf{r}, \Omega_{3}\right)}{\sqrt{P_{t}}} e^{i k_{t}\left(\Omega_{3}\right) x} \\
\mathbf{H}_{1}^{t}\left(\mathbf{r}, \Omega_{3}\right) & =\frac{\mathbf{h}_{t}\left(\mathbf{r}, \Omega_{3}\right)}{\sqrt{P_{t}}} e^{i k_{t}\left(\Omega_{3}\right) x}
\end{aligned}
$$

and

$$
\begin{aligned}
& \mathbf{E}_{2}^{f}\left(\mathbf{r}, \omega_{0}\right)=A_{f}(x) \frac{\mathbf{e}_{f}\left(\mathbf{r}, \omega_{0}\right)}{\sqrt{P_{f}}} e^{i k_{f}\left(\omega_{0}\right) x}, \\
& \mathbf{H}_{2}^{f}\left(\mathbf{r}, \omega_{0}\right)=A_{f}(x) \frac{\mathbf{h}_{f}\left(\mathbf{r}, \omega_{0}\right)}{\sqrt{P_{f}}} e^{i k_{f}\left(\omega_{0}\right) x}, \\
& \mathbf{E}_{2}^{t}\left(\mathbf{r}, \Omega_{3}\right)=A_{t}(x) \frac{\mathbf{e}_{t}\left(\mathbf{r}, \Omega_{3}\right)}{\sqrt{P_{t}}} e^{i k_{t}\left(\Omega_{3}\right) x}, \\
& \mathbf{H}_{2}^{t}\left(\mathbf{r}, \Omega_{3}\right)=A_{t}(x) \frac{\mathbf{h}_{t}\left(\mathbf{r}, \Omega_{3}\right)}{\sqrt{P_{t}}} e^{i k_{t}\left(\Omega_{3}\right) x},
\end{aligned}
$$

where $\Omega_{3}=3 \omega_{0}$ and $\mathbf{P}_{\mathrm{NL}}^{f}, \mathbf{P}_{\mathrm{NL}}^{t}$ can be derived from $\mathbf{P}_{\mathrm{NL}}(\mathbf{r}, t)=\hat{\chi}^{(3)}(\mathbf{r}, t): \mathbf{E}(\mathbf{r}, t) \mathbf{E}(\mathbf{r}, t) \mathbf{E}(\mathbf{r}, t) \quad$ with $\quad \mathbf{E}(\mathbf{r}, t)=$ $\mathbf{E}_{2}^{f}(\mathbf{r}, t)+\mathbf{E}_{2}^{t}(\mathbf{r}, t)$ and $\hat{\chi}^{(3)}$ the third-order susceptibility tensor. After some simple algebra, the nonlinear polarizations at the fundamental and third-harmonic frequencies can be written as

$$
\begin{aligned}
& \mathbf{P}_{\mathrm{NL}}^{f}\left(\mathbf{r}, \omega_{0}\right) \\
& =\frac{3 A_{f}^{* 2}(x) A_{t}(x)}{P_{f} \sqrt{P_{t}}} \hat{\chi}^{(3)}: \mathbf{e}_{f}^{*}\left(\mathbf{r}, \omega_{0}\right) \mathbf{e}_{f}^{*}\left(\mathbf{r}, \omega_{0}\right) \mathbf{e}_{t}\left(\mathbf{r}, \Omega_{3}\right) e^{i\left(k_{t}-2 k_{f}\right) x},
\end{aligned}
$$$$
\mathbf{P}_{\mathrm{NL}}^{t}\left(\mathbf{r}, \Omega_{3}\right)
$$

$$
=\frac{A_{f}^{3}(x)}{P_{f} \sqrt{P_{f}}} \hat{\chi}^{(3)}: \mathbf{e}_{f}\left(\mathbf{r}, \omega_{0}\right) \mathbf{e}_{f}\left(\mathbf{r}, \omega_{0}\right) \mathbf{e}_{f}\left(\mathbf{r}, \omega_{0}\right) e^{3 i k_{f} x} .
$$


If we now consider the fields $\left(\mathbf{E}_{1}^{f}, \mathbf{H}_{1}^{f}, \mathbf{E}_{2}^{f}, \mathbf{H}_{2}^{f}, \mathbf{P}_{\mathrm{NL}}^{f}\right)$, the 1.h.s. of Eq. (16) gives

$$
\int_{-\infty}^{\infty} \nabla \cdot \mathbf{F} d y=\frac{3 i \omega_{0} A_{f}^{* 2} A_{t} e^{i \Delta k x}}{P_{f} \sqrt{P_{f} P_{t}}} \int_{-\infty}^{\infty} \mathbf{e}_{f}^{*} \cdot \hat{\chi}^{(3)}: \mathbf{e}_{f}^{*} \mathbf{e}_{f}^{*} \mathbf{e}_{t} d y,
$$

where $\Delta k=k_{t}\left(\Omega_{3}\right)-3 k_{f}\left(\omega_{0}\right)$, and the r.h.s. of Eq. (16) leads to

$$
\begin{aligned}
& \frac{\partial}{\partial x} \int_{-\infty}^{\infty} \mathbf{F} \cdot \hat{\mathbf{x}} d y=\frac{\partial}{\partial x} \int_{-\infty}^{\infty}\left(\mathbf{E}_{1}^{f *} \times \mathbf{H}_{2}^{f}+\mathbf{E}_{2}^{f} \times \mathbf{H}_{1}^{f *}\right) \cdot \hat{\mathbf{x}} d y \\
& \quad=\frac{d A_{f}(x)}{d x} \frac{1}{P_{f}} \int_{-\infty}^{\infty}\left(\mathbf{e}_{f}^{*} \times \mathbf{h}_{f}+\mathbf{e}_{f} \times \mathbf{h}_{f}^{*}\right) \cdot \hat{\mathbf{x}} d y=4 \frac{d A_{f}(x)}{d x}
\end{aligned}
$$

Comparing Eqs. (B4) and (B5), we obtain the coupled-mode equation for the slowly varying mode amplitude $A_{f}(x)$,

$$
\frac{d A_{f}(x)}{d x}=i \gamma_{f}^{(3)}(x) A_{f}^{* 2}(x) A_{t}(x) e^{i \Delta k x}
$$

where the nonlinear coefficient at the fundamental frequency is

$$
\begin{aligned}
& \gamma_{f}^{(3)}(x) \\
& \quad=\frac{3 \omega_{0}}{4 P_{f} \sqrt{P_{f} P_{t}}} \int_{-\infty}^{\infty} \mathbf{e}_{f}^{*} \cdot \hat{\chi}^{(3)}\left(\omega_{0} ;-\omega_{0},-\omega_{0}, \Omega_{3}\right) \vdots \mathbf{e}_{f}^{*} \mathbf{e}_{f}^{*} \mathbf{e}_{t} d y .
\end{aligned}
$$

Similarly, if we consider the fields $\left(\mathbf{E}_{1}^{t}, \mathbf{H}_{1}^{t}, \mathbf{E}_{2}^{t}, \mathbf{H}_{2}^{t}, \mathbf{P}_{\mathrm{NL}}^{t}\right)$, the 1.h.s. of Eq. (16) gives

$$
\begin{aligned}
\int_{-\infty}^{\infty} \nabla \cdot \mathbf{F} d y= & \frac{i \Omega_{3} A_{f}^{3} e^{i \Delta k x}}{P_{f} \sqrt{P_{f} P_{t}}} \\
& \times \int_{-\infty}^{\infty} \mathbf{e}_{t}^{*} \cdot \hat{\chi}^{(3)}\left(\Omega_{3} ; \omega_{0}, \omega_{0}, \omega_{0}\right) \vdots \mathbf{e}_{f} \mathbf{e}_{f} \mathbf{e}_{f} d y
\end{aligned}
$$

and the r.h.s. of Eq. (16) can be written as

$$
\begin{aligned}
& \frac{\partial}{\partial x} \int_{-\infty}^{\infty} \mathbf{F} \cdot \hat{\mathbf{x}} d y=\frac{\partial}{\partial x} \int_{-\infty}^{\infty}\left(\mathbf{E}_{1}^{t *} \times \mathbf{H}_{2}^{t}+\mathbf{E}_{2}^{t} \times \mathbf{H}_{1}^{t *}\right) \cdot \hat{\mathbf{x}} d y \\
& =\frac{d A_{t}(x)}{d x} \frac{1}{P_{t}} \int_{-\infty}^{\infty}\left(\mathbf{e}_{t}^{*} \times \mathbf{h}_{t}+\mathbf{e}_{t} \times \mathbf{h}_{t}^{*}\right) \cdot \hat{\mathbf{x}} d y=4 \frac{d A_{t}(x)}{d x}
\end{aligned}
$$

Finally, from Eqs. (B8) and (B9), we get the coupled-mode equation for the slowly varying amplitude $A_{t}(x)$ as

$$
\frac{d A_{t}(x)}{d x}=i \gamma_{t}^{(3)}(x) A_{f}^{3}(x) e^{-i \Delta k x}
$$

where the nonlinear coefficient at the third-harmonic frequency is

$\gamma_{t}^{(3)}(x)=\frac{\Omega_{3}}{4 P_{f} \sqrt{P_{f} P_{t}}} \int_{-\infty}^{\infty} \mathbf{e}_{t}^{*} \cdot \hat{\chi}^{(3)}\left(\Omega_{3} ; \omega_{0}, \omega_{0}, \omega_{0}\right): \mathbf{e}_{f} \mathbf{e}_{f} \mathbf{e}_{f} d y$.

We can also express the nonlinear coefficients in terms of the group indices and the effective third-order susceptibilities, $\chi_{\mathrm{eff}, f / t}^{(3)}(x)$, of the two interacting modes,

$$
\begin{aligned}
& \gamma_{f}^{(3)}(x)=\frac{12 \omega_{0}}{a \epsilon_{0}^{2} v_{g, f} \sqrt{v_{g, f} v_{g, t}}} \chi_{\mathrm{eff}, f}^{(3)}(x), \\
& \gamma_{t}^{(3)}(x)=\frac{4 \Omega_{3}}{a \epsilon_{0}^{2} v_{g, f} \sqrt{v_{g, f} v_{g, t}}} \chi_{\mathrm{eff}, t}^{(3)}(x),
\end{aligned}
$$

where

$$
\begin{aligned}
& \chi_{\mathrm{eff}, f}^{(3)}(x)=\frac{a^{3} \int_{-\infty}^{\infty} \mathbf{e}_{f}^{*} \cdot \hat{\chi}^{(3)}\left(\omega_{0} ;-\omega_{0},-\omega_{0}, \Omega_{3}\right) \mathbf{e}_{f}^{*} \mathbf{e}_{f}^{*} \mathbf{e}_{t} d y}{\left[\int_{A_{\text {cell }}}\left(\mathbf{e}_{f}^{*} \cdot \hat{\epsilon}_{r} \mathbf{e}_{f}+Z_{0}^{2} \mathbf{h}_{f}^{*} \cdot \hat{\mu}_{r} \mathbf{h}_{f}\right) d A\right]^{3 / 2}\left[\int_{A_{\text {cell }}}\left(\mathbf{e}_{t}^{*} \cdot \hat{\epsilon}_{r} \mathbf{e}_{t}+Z_{0}^{2} \mathbf{h}_{t}^{*} \cdot \hat{\mu}_{r} \mathbf{h}_{t}\right) d A\right]}, \\
& \chi_{\mathrm{eff}, t}^{(3)}(x)=\frac{a^{3} \int_{-\infty}^{\infty} \mathbf{e}_{t}^{*} \cdot \hat{\chi}^{(3)}\left(\Omega_{3} ; \omega_{0}, \omega_{0}, \omega_{0}\right): \mathbf{e}_{f} \mathbf{e}_{f} \mathbf{e}_{f} d y}{\left[\int_{A_{\text {cell }}}\left(\mathbf{e}_{f}^{*} \cdot \hat{\epsilon}_{r} \mathbf{e}_{f}+Z_{0}^{2} \mathbf{h}_{f}^{*} \cdot \hat{\mu}_{r} \mathbf{h}_{f}\right) d A\right]^{3 / 2}\left[\int_{A_{\text {cell }}}\left(\mathbf{e}_{t}^{*} \cdot \hat{\epsilon}_{r} \mathbf{e}_{t}+Z_{0}^{2} \mathbf{h}_{t}^{*} \cdot \hat{\mu}_{r} \mathbf{h}_{t}\right) d A\right]^{1 / 2}} .
\end{aligned}
$$

Introducing the averaged physical quantities,

$$
\begin{aligned}
\bar{\gamma}_{f, t}^{(3)} & =\frac{1}{a} \int_{x_{0}}^{x_{0}+a} \gamma_{f, t}^{(3)}(x) d x, \\
\bar{\gamma}_{\mathrm{eff}, f / t}^{(3)} & =\frac{1}{a} \int_{x_{0}}^{x_{0}+a} \gamma_{\mathrm{eff}, f / t}^{(3)}(x) d x,
\end{aligned}
$$

and then averaging Eqs. (B6) and (B10) over one lattice constant, we obtain the coupled-mode equations governing the nonlinear dynamics of the interacting modes,

$$
\begin{aligned}
& \frac{d \bar{A}_{f}(x)}{d x}=i \bar{\gamma}_{f}^{(3)} \bar{A}_{f}^{* 2}(x) \bar{A}_{t}(x) e^{i \Delta k x}, \\
& \frac{d \bar{A}_{t}(x)}{d x}=i \bar{\gamma}_{t}^{(3)} \bar{A}_{f}^{3}(x) e^{-i \Delta k x},
\end{aligned}
$$



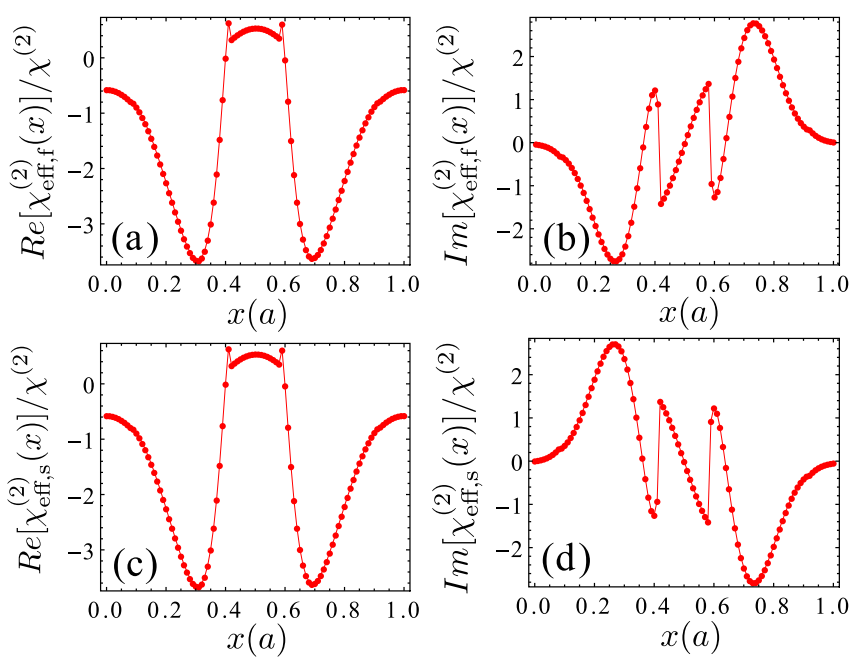

FIG. 7. (a, c) Real and (b, d) imaginary parts of $\chi_{\mathrm{eff}, f}^{(2)}(x)$ and $\chi_{\text {eff, }, s}^{(2)}(x)$ of Eq. (27) for SHG in one unit cell. While the real parts of $\chi_{\mathrm{eff}, f}^{(2)}(x)$ and $\chi_{\mathrm{eff}, s}^{(2)}(x)$ are even functions, with respect to the center of the unit cell, the imaginary parts of $\chi_{\mathrm{eff}, f}^{(2)}(x)$ and $\chi_{\mathrm{eff}, s}^{(2)}(x)$ are odd functions.

where $\bar{A}_{f}(x)$ and $\bar{A}_{t}(x)$ are the averaged mode amplitudes at the fundamental and third-harmonic frequencies, respectively.

\section{APPENDIX C: COMPARISON BETWEEN RIGOROUS NUMERICAL SIMULATIONS AND THE COUPLED-MODE THEORY}

In this Appendix, we present a computational analysis that illustrates how the coupled-mode theory derived above can be used to explain the full-wave dynamics obtained using COMSOL.

\section{Second-harmonic generation described by the coupled-mode theory}

The key quantities that characterize the coupled-mode theory describing second-harmonic generation are $\chi_{\mathrm{eff}, f}^{(2)}(x)$ and $\chi_{\text {eff, } s}^{(2)}(x)$, as defined by Eqs. (27a) and (27b), respectively. As we consider photonic crystals that are periodic in space, $\chi_{\mathrm{eff}, f}^{(2)}(x)$ and $\chi_{\mathrm{eff}, s}^{(2)}(x)$ are periodic functions of $x$, and we only need to show their $x$ dependence in one unit cell. Thus, in Fig. 7 we depict the $x$ dependence of $\chi_{\mathrm{eff}, f}^{(2)}(x)$ and $\chi_{\mathrm{eff}, s}^{(2)}(x)$ in one unit cell. As one can see, while the real parts of $\chi_{\mathrm{eff}, f}^{(2)}(x)$ and $\chi_{\text {eff,s }}^{(2)}(x)$ are even functions, with respect to the center of the unit cell, the imaginary parts of $\chi_{\mathrm{eff}, f}^{(2)}(x)$ and $\chi_{\mathrm{eff}, s}^{(2)}(x)$ are odd functions. In fact, it can be easily demonstrated that $\chi_{\mathrm{eff}, f}^{(2)}(x)=\chi_{\mathrm{eff}, s}^{(2)} *(x)$. Therefore, the averages of $\chi_{\mathrm{eff}, f}^{(2)}(x)$ and $\chi_{\mathrm{eff}, s}^{(2)}(x)$ in one unit cell are real numbers and are equal to each other.

After one computes $\bar{\gamma}_{f, s}^{(2)}$ and $\bar{\chi}_{\text {eff, } f / s}^{(2)}$ by averaging $\gamma_{f, s}^{(2)}(x)$ and $\chi_{\text {eff, } f / s}^{(2)}(x)$, respectively, in one unit cell, one can straightforwardly solve the coupled-mode equations expressed as Eqs. (29). Thus, we present in Fig. 8 the evolution of the generated second-harmonic wave as a function of the

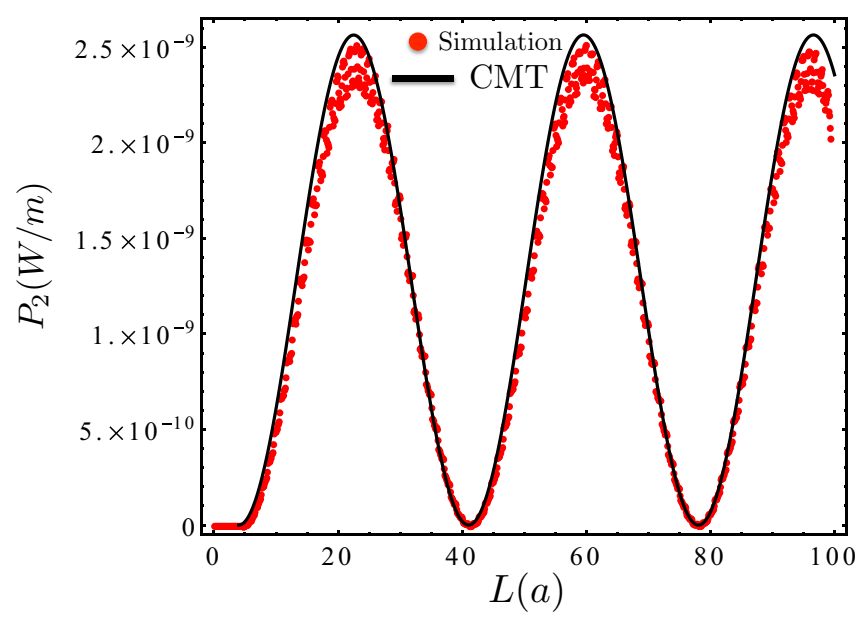

FIG. 8. Evolution of the power of the second-harmonic wave as a function of the propagation distance. Red circles are from rigorous full-wave numerical simulations, while the black curve is obtained from solving the coupled-mode equations expressed as Eqs. (29).

propagation distance. It is shown in this figure that there is a good agreement between the coupled-mode theory and rigorous numerical simulations with regard to both the oscillation period and the amplitude of the power of the second-harmonic wave.

\section{Third-harmonic generation described by the coupled-mode theory}

Similarly to the case of SHG, the key quantities that characterize the coupled-mode theory of third-harmonic generation are $\chi_{\text {eff, } f}^{(3)}(x)$ and $\chi_{\text {eff, } t}^{(3)}(x)$, as defined by Eqs. (B13). Both these physical quantities are periodic functions of the $x$ coordinate, their $x$ dependence being presented in Fig. 9. Also similarly to the case of SHG, while the real parts of $\chi_{\text {eff, } f}^{(3)}(x)$ and
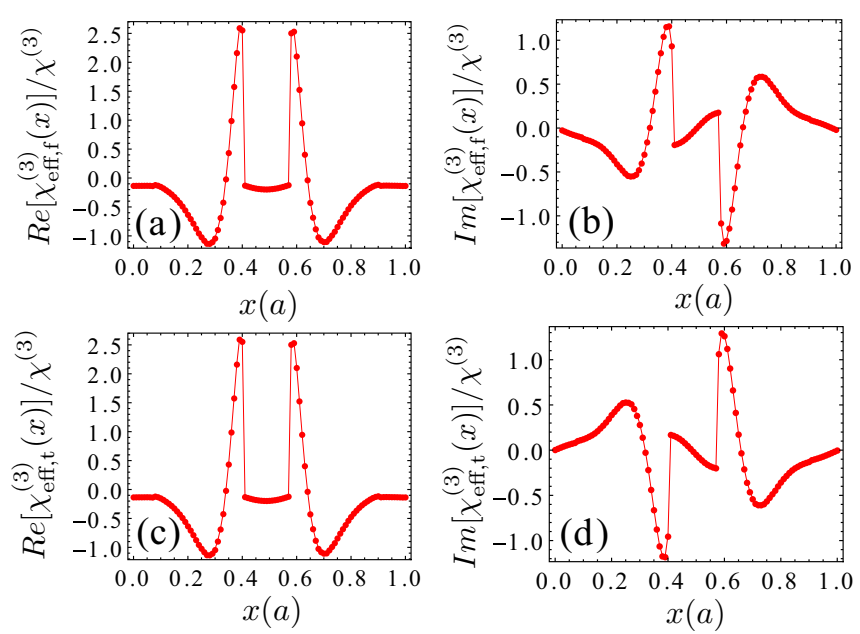

FIG. 9. (a, c) Real and (b, d) imaginary parts of $\chi_{\mathrm{eff}, f}^{(3)}(x)$ and $\chi_{\mathrm{eff}, t}^{(3)}(x)$ of Eqs. (B13) for THG in one unit cell. Similarly to the case of SHG, while the real parts of $\chi_{\mathrm{eff}, f}^{(3)}(x)$ and $\chi_{\mathrm{eff}, t}^{(3)}(x)$ are even functions, the imaginary parts of $\chi_{\mathrm{eff}, f}^{(3)}(x)$ and $\chi_{\mathrm{eff}, t}^{(3)}(x)$ are odd functions. 


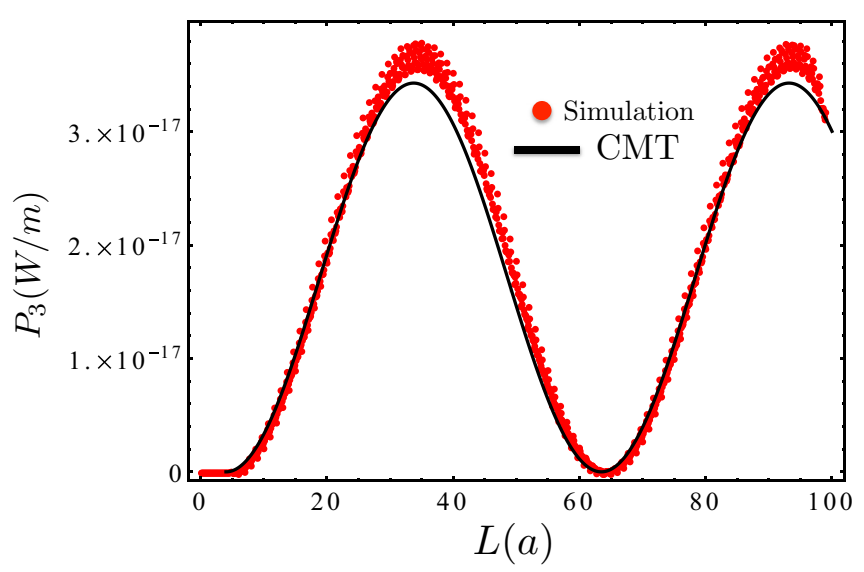

FIG. 10. Evolution of the power of the third-harmonic wave vs. the propagation distance. Red circles are from numerical simulations, while the black curve is from solving Eqs. (B15).

$\chi_{\mathrm{eff}, t}^{(3)}(x)$ are even functions, the imaginary parts of $\chi_{\mathrm{eff}, f}^{(3)}(x)$ and $\chi_{\mathrm{eff}, t}^{(3)}(x)$ are odd functions. Therefore, the averages of $\chi_{\mathrm{eff}, f}^{(3)}(x)$ and $\chi_{\mathrm{eff}, t}^{(3)}(x)$ in one unit cell are real numbers and are equal to each other.

We have solved Eqs. (B15) using $\bar{\gamma}_{f, t}^{(3)}$ obtained by averaging $\gamma_{f, t}^{(3)}(x)$ in one unit cell and present the results in Fig. 10. We again find a good agreement between the coupled-mode theory and rigorous numerical simulations regarding both the oscillation period and the amplitude of the power of the thirdharmonic wave.

\section{Coupled-mode theory of slow-light nonlinearity enhancement}

The group index $n_{g}$ of the edge modes in a generic case, e.g., Fig. 4, is limited to 5-7. However, we have shown in the text that the shape of the dispersion curve of the one-way
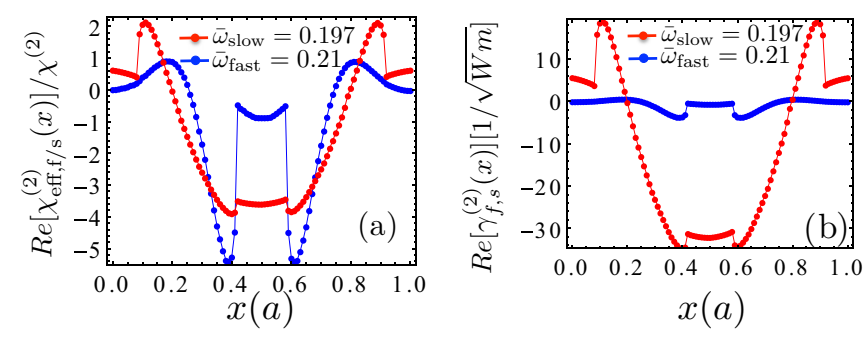

FIG. 11. (a) $\chi_{\mathrm{eff}, f / s}^{(2)}(x)$ defined by Eqs. (27a) and (27b) for SHG in one unit cell at two frequencies, $\bar{\omega}_{\text {slow }}=0.197$ and $\bar{\omega}_{\text {fast }}=0.21$, where the group indices of the fundamental wave at the two frequencies are $n_{g}^{f}\left(\bar{\omega}_{\text {slow }}\right) \simeq 174$ and $n_{g}^{f}\left(\bar{\omega}_{\text {fast }}\right) \simeq 12\left[n_{g}^{s}\left(\bar{\omega}_{\text {slow }}\right) \simeq 5\right.$ and $\left.n_{g}^{s}\left(\bar{\omega}_{\text {fast }}\right) \simeq 4\right]$. (b) The nonlinear coefficient $\gamma_{f / s}^{(2)}(x)$ of Eqs. (21) and (25) in one unit cell at the two frequencies $\bar{\omega}_{\text {slow }}$ and $\bar{\omega}_{\text {fast }}$. One can see the enhancement of the nonlinear coefficient due to the slow-light effect $\left[n_{g}^{f}\left(\bar{\omega}_{\text {slow }}\right) / n_{g}^{f}\left(\bar{\omega}_{\text {fast }}\right) \simeq 15\right.$, whereas $\bar{\gamma}_{f, s}^{(2)}\left(\bar{\omega}_{\text {slow }}\right) / \bar{\gamma}_{f, s}^{(2)}\left(\bar{\omega}_{\text {fast }}\right) \simeq 18$ according to Eq. (28)].

edge modes can be tailored, leading to much larger $n_{g}$, as per Fig. 5. As is well known, this will enhance the efficiency of the nonlinear process. We plot in Fig. 11(a) the function $\chi_{\text {eff }, f / s}^{(2)}(x)$ at two frequencies, $\bar{\omega}_{\text {slow }}=0.197$ and $\bar{\omega}_{\text {fast }}=0.21$, with $n_{g}^{f}\left(\bar{\omega}_{\text {slow }}\right) \simeq 174$ and $n_{g}^{f}\left(\bar{\omega}_{\text {fast }}\right) \simeq 12\left[n_{g}^{s}\left(\bar{\omega}_{\text {slow }}\right) \simeq 5\right.$ and $n_{g}^{s}\left(\bar{\omega}_{\text {fast }}\right) \simeq 4$ have similar values at these two frequencies]. While the amplitudes of $\chi_{\mathrm{eff}, f / s}^{(2)}(x)$ at these two frequencies are comparable, from Fig. 11(b), which shows the nonlinear coefficient $\gamma_{f / s}^{(2)}(x)$ of Eqs. (21) and (25), one can see that $\gamma_{f / s}^{(2)}(x)$ at $\bar{\omega}_{\text {slow }}$ is significantly enhanced compared to $\gamma_{f / s}^{(2)}(x)$ at $\bar{\omega}_{\text {fast }}$. The ratio of the averaged nonlinear coefficient in one unit cell according to Eq. (28) and Fig. 5(b) is $\bar{\gamma}_{f, s}^{(2)}\left(\bar{\omega}_{\text {slow }}\right) / \bar{\gamma}_{f, s}^{(2)}\left(\bar{\omega}_{\text {fast }}\right) \simeq$ 18 , which roughly agrees with $n_{g}^{f}\left(\bar{\omega}_{\text {slow }}\right) / n_{g}^{f}\left(\bar{\omega}_{\text {fast }}\right) \simeq 15$. This enhancement of the nonlinear coefficient leads to the enhancement of the efficiency of SHG [see, e.g., Fig. 5(c)].
[1] M. Z. Hasan and C. L. Kane, Colloquium: Topological insulators, Rev. Mod. Phys. 82, 3045 (2010).

[2] X.-L. Qi and S.-C. Zhang, Topological insulators and superconductors, Rev. Mod. Phys. 83, 1057 (2011).

[3] L. Lu, J. D. Joannopoulos, and M. Soljacic, Topological photonics, Nat. Photon. 8, 821 (2014).

[4] A. B. Khanikaev and G. Shvets, Two-dimensional topological photonics, Nat. Photon. 11, 763 (2017).

[5] X.-C. Suna, C. He, X.-P. Liu, M.-H. Lu, S.-N. Zhu, and Y.-F. Chen, Two-dimensional topological photonic systems, Prog. Quantum Electron. 55, 52 (2017).

[6] Y. Wu, C. Li, X. Hu, Y. Ao, Y. Zhao, and Q. Gong, Applications of topological photonics in integrated photonic devices, Adv. Optical Mater. 5, 1700357 (2017).

[7] B.-Y. Xie, H.-F. Wang, X.-Y. Zhu, M.-H. Lu, Z. D. Wang, and Y.-F. Chen, Photonics meets topology, Opt. Express 26, 24531 (2018).

[8] M. S. Rider, S. J. Palmer, S. R. Pocock, X. Xiao, P. A. Huidobro, and V. Giannini, A perspective on topological nanophotonics:
Current status and future challenges, J. Appl. Phys. 125, 120901 (2019).

[9] T. Ozawa, H. M. Price, A. Amo, N. Goldman, M. Hafezi, L. Lu, M. C. Rechtsman, D. Schuster, J. Simon, O. Zilberberg, and I. Carusotto, Topological photonics, Rev. Mod. Phys. 91, 015006 (2019).

[10] F. D. M. Haldane and S. Raghu, Possible Realization of Directional Optical Waveguides in Photonic Crystals with Broken Time-Reversal Symmetry, Phys. Rev. Lett. 100, 013904 (2008).

[11] G. D. Nittis and M. Lein, On the role of symmetries in the theory of photonic crystals, Ann. Phys. 350, 568 (2014).

[12] L. Feng, R. EI-Ganainy, and L. Ge, Non-Hermitian photonics based on parity-time symmetry, Nat. Photon. 11, 752 (2017).

[13] G. Harari, M. A. Bandres, Y. Lumer, M. C. Rechtsman, Y. D. Chong, M. Khajavikhan, D. N. Christodoulides, and M. Segev, Topological insulator laser: Theory, Science 359, eaar4003 (2018).

[14] M. A. Bandres, S. Wittek, G. Harari, M. Parto, J. Ren, M. Segev, D. N. Christodoulides, and M. Khajavikhan, Topological insulator laser: Experiments, Science 359, eaar4005 (2018). 
[15] R. W. Boyd, Nonlinear Optics, 3rd ed. (Academic Press, San Diego, CA, 2008).

[16] Y. Lumer, Y. Plotnik, M. C. Rechtsman, and M. Segev, SelfLocalized States in Photonic Topological Insulators, Phys. Rev. Lett. 111, 243905 (2013).

[17] D. Leykam and Y. D. Chong, Edge Solitons in NonlinearPhotonic Topological Insulators, Phys. Rev. Lett. 117, 143901 (2016)

[18] C. Qian, K. H. Choi, R. P. H. Wu, Y. Zhang, K. Guo, and K. H. Fung, Nonlinear frequency up-conversion via double topological edge modes, Opt. Express 26, 5083 (2018).

[19] Y. Wang, L.-J. Lang, C. H. Lee, B. Zhang, and Y. D. Chong, Topologically enhanced harmonic generation in a nonlinear transmission line metamaterial, Nat. Commun. 10, 1102 (2019).

[20] S. Kruk, A. Poddubny, D. Smirnova, L. Wang, A. Slobozhanyuk, A. Shorokhov, I. Kravchenko, B. L. Davies, and Y. S. Kivshar, Nonlinear light generation in topological nanostructures, Nat. Nanotechnol. 14, 126 (2019).

[21] V. Peano, M. Houde, F. Marquardt, and A. A. Clerk, Topological quantum fluctuations and traveling wave amplifiers, Phys. Rev. X 6, 041026 (2016).

[22] S. Mittal, E. A. Goldschmidt, and M. Hafezi, A topological source of quantum light, Nature 561, 502 (2018).

[23] D. A. Dobrykh, A. V. Yulin, A. P. Slobozhanyuk, A. N. Poddubny, and Y. Kivshar, Nonlinear Control of Electromagnetic Topological Edge States, Phys. Rev. Lett. 121, 163901 (2018).

[24] D. Smirnova, S. Kruk, D. Leykam, E. M.-Gaykazyan, D.-Y. Choi, and Y. Kivshar, Third-Harmonic Generation in Photonic Topological Metasurfaces, Phys. Rev. Lett. 123, 103901 (2019).

[25] J. D. Joannopoulos, S. G. Johnson, J. N. Winn, and R. D. Meade, Photonic Crystals: Molding the Flow of Light, 2nd ed. (Princeton University Press, Princeton, NJ, 2008).

[26] COMSOL Multiphysics; www.comsol.com.

[27] BandSOLVE; www.synopsys.com.

[28] Z. Wang, Y. D. Chong, J. D. Joannopoulos, and M. Soljacic, Reflection-Free One-Way Edge Modes in a Gyromagnetic Photonic Crystal, Phys. Rev. Lett. 100, 013905 (2008).

[29] Z. Wang, Y. Chong, J. D. Joannopoulos, and M. Soljacic, Observation of unidirectional backscattering-immune topological electromagnetic states, Nature 461, 772 (2009).

[30] Y. Poo, R.-X. Wu, Z. Lin, Y. Yang, and C. T. Chan, Experimental Realization of Self-Guiding Unidirectional Electromagnetic Edge States, Phys. Rev. Lett. 106, 093903 (2011).

[31] S. A. Skirlo, L. Lu, Y. Igarashi, Q. Yan, J. Joannopoulos, and M. Soljacic, Experimental Observation of Large Chern Numbers in Photonic Crystals, Phys. Rev. Lett. 115, 253901 (2015).

[32] B. Bahari, A. Ndao, F. Vallini, A. E. Amili, Y. Fainman, and B. Kante, Nonreciprocal lasing in topological cavities of arbitrary geometries, Science 358, 636 (2017).

[33] F.-F. Li, H.-X. Wang, Z. Xiong, Q. Lou, P. Chen, R.-X. Wu, Y. Poo, J.-H. Jiang, and S. John, Topological light-trapping on a dislocation, Nat. Commun. 9, 2462 (2018).

[34] T. Fukui, Y. Hatsugai, and H. Suzuki, Chern numbers in discretized Brillouin zone: Efficient method of computing (spin) Hall conductances, J. Phys. Soc. Jpn. 74, 1674 (2005).

[35] N. C. Panoiu, J. F. McMillan, and C. W. Wong, Theoretical analysis of pulse dynamics in silicon photonic crystal wire waveguides, IEEE J. Select. Top. Quantum Electron. 16, 257 (2010).
[36] S. Lavdas and N. C. Panoiu, Theory of pulsed four-wave mixing in one-dimensional silicon photonic crystal slab waveguides, Phys. Rev. B 93, 115435 (2016).

[37] A. W. Snyder and J. Love, Optical Waveguide Theory (Chapman \& Hall, London, 1983).

[38] B. E. A. Saleh and M. C. Teich, Fundamentals of Photonics. Wiley Series in Pure and Applied Optics, 2nd rev. ed. (WileyBlackwell, Hoboken, NJ, 2007).

[39] G. D. Boyd, T. J. Bridges, M. A. Pollack, and E. H. Turner, Microwave Nonlinear Susceptibilities Due to Electronic and Ionic Anharmonicities in Acentric Crystals, Phys. Rev. Lett. 26, 387 (1971).

[40] T. J. Bridges and A. R. Strand, Submillimeter wave generation by difference-frequency mixing in GaAs, Appl. Phys. Lett. 20, 382 (1972).

[41] G. D. Boyd, T. J. Bridges, C. K. N. Patel, and E. Buehler, Phase-matched submillimeter wave generation by differencefrequency mixing in $\mathrm{ZnGeP}_{2}$, Appl. Phys. Lett. 21, 553 (1972).

[42] B. H. Ahn, W. W. Clark, R. R. Shurtz, and C. D. Bates, Second harmonic generation in $\mathrm{LiNbO}_{3}$ and $\mathrm{LITaO}_{3}$ in the millimeter wave region, J. Appl. Phys. 54, 1251 (1983).

[43] D. M. Pozar, Microwave Engineering, 4th ed. (John Wiley \& Sons, New York, 2011).

[44] M. C. Rechtsman, J. M. Zeuner, Y. Plotnik, Y. Lumer, D. Podolsky, F. Dreisow, S. Nolte, M. Segev, and A. Szameit, Photonic floquet topological insulators, Nature 496, 196 (2013).

[45] M. Hafezi, S. Mittal, J. Fan, A. Migdall, and J. M. Taylor, Imaging topological edge states in silicon photonics, Nat. Photon. 7, 1001 (2013).

[46] J. Noh, S. Huang, K. P. Chen, and M. C. Rechtsman, Observation of Photonic Topological Valley Hall Edge States, Phys. Rev. Lett. 120, 063902 (2018).

[47] D. R. Smith, W. J. Padilla, D. C. Vier, S. C. Nemat-Nasser, and S. Schultz, Composite Medium with Simultaneously Negative Permeability and Permittivity, Phys. Rev. Lett. 84, 4184 (2000).

[48] M. W. Klein, C. Enkrich, M. Wegener, and S. Linden, Secondharmonic generation from magnetic metamaterials, Science 313, 502 (2006).

[49] M. Lapine, I. V. Shadrivov, and Y. S. Kivshar, Colloquium: Nonlinear metamaterials, Rev. Mod. Phys. 86, 1093 (2013).

[50] W. Gao, M. Lawrence, B. Yang, F. Liu, F. Fang, B. Beri, J. Li, and S. Zhang, Topological Photonic Phase in Chiral Hyperbolic Metamaterials, Phys. Rev. Lett. 114, 037402 (2015).

[51] K. Y. M. Yeung, J. Chee, H. Yoon, Y. Song, J. Kong, and D. Ham, Far-infrared graphene plasmonic crystals for plasmonic band engineering, Nano Lett. 14, 2479 (2014).

[52] D. Jin, T. Christensen, M. Soljacic, N. X. Fang, L. Lu, and X. Zhang, Infrared Topological Plasmons in Graphene, Phys. Rev. Lett. 118, 245301 (2017).

[53] D. Pan, R. Yu, H. Xu, and F. J. G. de Abajo, Topologically protected Dirac plasmons in a graphene superlattice, Nat. Commun. 8, 1243 (2017).

[54] J. W. You, Z. Lan, and N. C. Panoiu, Four-wave mixing of topological edge plasmons in graphene metasurfaces, Sci. Adv. 6, eaaz3910 (2020).

[55] M. Hafezi, E. A. Demler, M. D. Lukin and J. M. Taylor, Robust optical delay lines with topological protection, Nat. Phys. 7, 907 (2011).

[56] M. I. Shalaev, W. Walasik, A. Tsukernik, Y. Xu and N. M. Litchinitser, Robust topologically protected transport 
in photonic crystals at telecommunication wavelengths, Nat. Nanotechnol. 14, 31 (2019).

[57] X.-T. He, E.-T. Liang, J.-J. Yuan, H.-Y. Qiu, X.-D. Chen, F.-L. Zhao, and J.-W. Dong, A silicon-on-insulator slab for topological valley transport, Nat. Commun. 10, 872 (2019).

[58] Q. Chen, L. Zhang, M. He, Z. Wang, X. Lin, F. Gao, Y. Yang, B. Zhang and H. Chen, Valley-Hall photonic topological insulators with dual-band kink states, Adv. Opt. Mater. 7, 1900036 (2019).

[59] L.-H. Wu and X. Hu, Scheme for Achieving a Topological Photonic Crystal by Using Dielectric Material, Phys. Rev. Lett. 114, 223901 (2015).

[60] S. Barik, A. Karasahin, C. Flower, T. Cai, H. Miyake, W. DeGottardi, M. Hafezi, and E. Waks, A topological quantum optics interface, Science 359, 666 (2018).

[61] S. Peng, N. J. Schilder, X. Ni, J. van de Groep, M. L. Brongersma, A. Alu, A. B. Khanikaev, H. A. Atwater, and A. Polman, Probing the Band Structure of Topological Silicon Photonic Lattices in the Visible Spectrum, Phys. Rev. Lett. 122, 117401 (2019).

[62] J. Noh, W. A. Benalcazar, S. Huang, M. J. Collins, K. P. Chen, T. L. Hughes and M. C. Rechtsman, Topological protection of photonic mid-gap defect modes, Nat. Photon. 12, 408 (2018).

[63] S. Mittal, V. V. Orre, D. Leykam, Y. D. Chong, and M. Hafezi, Photonic Anomalous Quantum Hall Effect, Phys. Rev. Lett. 123, 043201 (2019)

[64] T. Shi, H. J. Kimble, and J. I. Cirac, Topological phenomena in classical optical networks, Proc. Natl. Acad. Sci. USA 114, E8967 (2017).

[65] S. A. Skirlo, L. Lu, and M. Soljacic, Multimode One-Way Waveguides of Large Chern Numbers, Phys. Rev. Lett. 113, 113904 (2014).

[66] M. Kauranen and A. V. Zayats, Nonlinear plasmonics, Nat. Photon. 6, 737 (2012).

[67] N. C. Panoiu, W. Sha, D. Y. Lei, and G. C. Li, Nonlinear optics in plasmonic nanostructures, J. Opt. 20, 083001 (2018).
[68] Z. Yang, F. Gao, X. Shi, X. Lin, Z. Gao, Y. Chong, and B. Zhang, Topological Acoustics, Phys. Rev. Lett. 114, 114301 (2015).

[69] A. Bojahr, M. Gohlke, W. Leitenberger, J. Pudell, M. Reinhardt, A. von Reppert, M. Roessle, M. Sander, P. Gaal, and M. Bargheer, Second Harmonic Generation of Nanoscale Phonon Wave Packets, Phys. Rev. Lett. 115, 195502 (2015).

[70] H. Ge, M. Yang, C. Ma, M.-H. Lu, Y.-F. Chen, N. Fang, and P. Sheng, Breaking the barriers: Advances in acoustic functional materials, Natl. Sci. Rev. 5, 159 (2018).

[71] A. V. Chumak, V. I. Vasyuchka, A. A. Serga, and B. Hillebrands, Magnon spintronics, Nat. Phys. 11, 453 (2015).

[72] T. Sebastian, T. Bracher, P. Pirro, A. A. Serga, B. Hillebrands, T. Kubota, H. Naganuma, M. Oogane, and Y. Ando, Nonlinear Emission of Spin-Wave Caustics from an Edge Mode of a Microstructured $\mathrm{Co}_{2} \mathrm{Mn}_{0.6} \mathrm{Fe}_{0.4} \mathrm{Si}$ Waveguide, Phys. Rev. Lett. 110, 067201 (2013).

[73] X. S. Wang, H. W. Zhang, and X. R. Wang, Topological magnonics: A paradigm for spin-wave manipulation and device design, Phys. Rev. Appl. 9, 024029 (2018).

[74] T. Karzig, C.-E. Bardyn, N. H. Lindner, and G. Refael, Topological polaritons, Phys. Rev. X 5, 031001 (2015).

[75] S. Klembt, T. H. Harder, O. A. Egorov, K. Winkler, R. Ge, M. A. Bandres, M. Emmerling, L. Worschech, T. C. H. Liew, M. Segev, C. Schneider, and S. Hofling, Exciton-polariton topological insulator, Nature 562, 552 (2018).

[76] W. Warkentin, J. Mund, D. R. Yakovlev, V. V. Pavlov, R. V. Pisarev, A. V. Rodina, M. A. Semina, M. M. Glazov, E. L. Ivchenko, and M. Bayer, Third harmonic generation on excitonpolaritons in bulk semiconductors subject to a magnetic field, Phys. Rev. B 98, 075204 (2018).

[77] P. Knuppel, S. Ravets, M. Kroner, S. Falt, W. Wegscheider, and A. Imamoglu, Nonlinear optics in the fractional quantum Hall regime, Nature 572, 91 (2019). 